Article

\title{
Radiative Recombination and Photoionization Data for Tungsten Ions. Electron Structure of Ions in Plasmas
}

\author{
Malvina B. Trzhaskovskaya ${ }^{1, *}$ and Vladimir K. Nikulin ${ }^{2}$ \\ ${ }^{1}$ Petersburg Nuclear Physics Institute, Gatchina, Leningrad District, 188300, Russia \\ ${ }^{2}$ Ioffe Physical Technical Institute, St. Petersburg 194021, Russia; \\ E-Mail: nikulin@astro.ioffe.ru \\ * Author to whom correspondence should be addressed; E-Mail: Trzhask@MT5605.spb.edu \\ Academic Editor: Alfred Müller and Bastiaan J. Braams
}

Received: 24 February 2015 / Accepted: 6 May 2015 / Published: 18 May 2015

\begin{abstract}
Theoretical studies of tungsten ions in plasmas are presented. New calculations of the radiative recombination and photoionization cross-sections, as well as radiative recombination and radiated power loss rate coefficients have been performed for 54 tungsten ions for the range $\mathrm{W}^{6+}-\mathrm{W}^{71+}$. The data are of importance for fusion investigations at the reactor ITER, as well as devices ASDEX Upgrade and EBIT. Calculations are fully relativistic. Electron wave functions are found by the Dirac-Fock method with proper consideration of the electron exchange. All significant multipoles of the radiative field are taken into account. The radiative recombination rates and the radiated power loss rates are determined provided the continuum electron velocity is described by the relativistic Maxwell-Jüttner distribution. The impact of the core electron polarization on the radiative recombination cross-section is estimated for the Ne-like iron ion and for highly-charged tungsten ions within an analytical approximation using the Dirac-Fock electron wave functions. The effect is shown to enhance the radiative recombination cross-sections by $\lesssim 20 \%$. The enhancement depends on the photon energy, the principal quantum number of polarized shells and the ion charge. The influence of plasma temperature and density on the electron structure of ions in local thermodynamic equilibrium plasmas is investigated. Results for the iron and uranium ions in dense plasmas are in good agreement with previous calculations. New calculations were performed for the tungsten ion in dense plasmas on the basis of the average-atom model, as well as for the impurity tungsten ion in fusion plasmas using the non-linear self-consistent field screening model. The temperature and density dependence of the ion charge, level energies and populations are considered.
\end{abstract}


Keywords: radiative recombination; photoionization; rate coefficient; Dirac-Fock method; tungsten ion; polarization effect; density; temperature

\section{Introduction}

Experimental and theoretical investigation of tungsten becomes important due to its employment in up-to-date tokamaks. In fusion reactors, the core plasma temperature is expected to be about $25 \mathrm{keV}$. Tungsten is used as a wall material for the divertor in JET and for the first-wall in ASDEX Upgrade. It is assumed that tungsten will be used as a plasma-facing material in the fusion reactor ITER due to such features as high thermal conductivity, a high melting point, low erosion, low sputtering and low tendency to trap tritium [1-3]. However, tungsten as a high-Z plasma impurity is an efficient radiator at high temperature, and the tungsten influx should be controlled. Because of this, the tungsten radiative characteristics have become the subject of study. Radiative recombination (RR) of tungsten impurity ions with plasma electrons and the inverse process of photoionization of tungsten ions are significant mechanisms that influence the ionization equilibria and the thermal balance of fusion plasmas. Tungsten is also investigated for such devices as ASDEX Upgrade and EBIT to produce data for ITER. In particular, the new active spectroscopy diagnostic of highly-charged ions is applied in studies at EBIT [4]. The diagnostic involves accurate theoretical values of the RR cross-sections for different tungsten ions. However, until recently, the systematic theoretical data on ionization-recombination cross-sections and coefficients for heavy-element ions have been practically unavailable.

Therefore, our purpose was to elaborate a new database, including accurate values of partial and total RR cross-sections (RRCS), partial photoionization cross-sections (PCS), partial and total RR rate coefficients (RR rates) and radiated power loss rate coefficients (RPL rates) for the majority of tungsten ions. We have performed fully relativistic calculations of RRCS and PCS using the Dirac-Fock (DF) method taking into account all significant multipoles of the radiative field for a large number of the heavy element impurity ions, among which are tungsten ions with closed shells, namely, $\mathrm{W}^{6+}, \mathrm{W}^{28+}$, $\mathrm{W}^{38+}, \mathrm{W}^{46+}, \mathrm{W}^{56+}, \mathrm{W}^{64+}$, as well as $\mathrm{W}^{72+}-\mathrm{W}^{74+}$ [5]. Then, calculations of the RR rates for these highly-charged tungsten ions, with the exception of $\mathrm{W}^{6+}$, have been carried out in a wide temperature range [6]. Analysis of data for tungsten ions and a comparison of our results with available previous calculations are described in [7]. Numerical results of RPL rates for the eight highly-charged tungsten ions are also given in [7].

In the framework of the IAEA Coordinated Research Project (CRP) "Spectroscopic and Collisional Data for Tungsten in Plasma from $1 \mathrm{eV}$ to $20 \mathrm{keV}$ ", we performed new calculations of the RR and photoionization data for additional 54 tungsten ions in the charge range $\mathrm{W}^{6+}-\mathrm{W}^{71+}$ [8-10]. Accurate relativistic values of the partial and total RRCS, partial PCS, as well as partial and total RR/RPL rates were obtained. Total RRCS were calculated in the electron energy range from $1 \mathrm{eV}-\sim 80 \mathrm{keV}$. Partial PCS and RRCS were fitted by an analytical expression with five fit parameters in the wide photon energy range for all electron states with principal quantum numbers $n \leq 10$ and orbital momenta $\ell \leq 4$. Partial $\mathrm{RR}$ and RPL rates for the same states and the associated total rates are presented for eleven values of temperature in the range from $10^{4} \mathrm{~K}-10^{9} \mathrm{~K}$. Values of RR and RPL rates for $\mathrm{W}^{6+}$ are given in this paper 
(see Tables A1 and A2 in Appendix). Now, the part of our database concerning tungsten contains data for 62 ions from the range $\mathrm{W}^{6+}-\mathrm{W}^{74+}$. The results were added to our extended unified database containing the RR and photoionization data for about 170 heavy element impurity ions occurring in fusion plasmas. New data were included in the IAEA Atomic and Molecular database [11].

With the context of CRP, we estimated also an impact of the target core electron polarization following the RR process on RRCS outside the regions of the dielectronic recombination resonances, i.e., the so-called polarization RR effect (PRR effect). Calculations were carried out in the framework of the analytical "stripping" approximation using the relativistic DF electron wave functions. The approximation was shown to provide a reasonable estimation of the PRR effect.

The effect was considered for the Fe XVII ion where a comparison with experimental data was performed [12]. The experimental values of the electron-impact excitation cross-section (EIECS) were determined in [13] by normalizing to measured intensities of the RR peaks, which were independently normalized to the associated theoretical RRCS. The experimental EIECS turned out to be lower by $~ 25 \%$ as compared to all available theoretical values. We showed that the problem with the determination of absolute values of the measured EIECS was that only the RR channel was taken into account in theoretical RRCS used for normalization, while the PRR channel was overlooked. The inclusion of the PRR channel eliminates this puzzling discrepancy between experimental and theoretical EIECS.

Then, the PRR effect was assessed for the tungsten highly-charged ions [14]. It was obtained that enhancement factor $F_{n}$ changes from $\sim 15 \%$ to $\lesssim 1 \%$. We showed that the factor $F_{n}$ depends on the photon energy, the principal quantum number of polarized shells and the ion charge, but is practically independent of the final electron state in the RR process.

We studied also an electron structure of ions in local thermodynamic equilibrium (LTE) plasmas [15]. The influence of plasma temperature and density on the energy spectrum and level populations of an ion in dense and fusion plasmas was considered. The code PLASMASATOM was designed on the basis of our computer program complex RAINE (Relativistic atom. Interaction of electromagnetic radiation and nucleus with atomic electrons) [16-18]. The electron wave functions were calculated by the Dirac-Slater (DS) method with approximate consideration for the electron exchange. The code is of the type of the Los Alamos INFERNO code [19,20] and PURGATORIOcode [21], as well as more advanced PARADISIO code [22]. Our results concerning the ionization charges of iron and uranium ions are shown to be in a good agreement with data of previous calculations. New results were obtained for the impurity tungsten ion in fusion plasmas at low temperatures using the non-linear self-consistent field screening model and for the dense tungsten plasmas in the wide temperature range using the average-atom model.

\section{Radiative Recombination and Photoionization Data}

\subsection{Method of Calculations}

The exact relativistic treatment of the photoionization process having regard to all multipoles of the radiative field leads to the following expressions for PCS in the i-th atomic subshell [5]: 


$$
\begin{aligned}
\sigma_{\mathrm{ph}}^{(i)} & =\frac{4 \pi^{2} \alpha}{k\left(2 j_{i}+1\right)} \sum_{L} \sum_{\kappa}\left[(2 L+1) Q_{L L}^{2}(\kappa)+L Q_{L+1 L}^{2}(\kappa)\right. \\
& \left.+(L+1) Q_{L-1 L}^{2}(\kappa)-2 \sqrt{L(L+1)} Q_{L-1 L}(\kappa) Q_{L+1 L}(\kappa)\right]
\end{aligned}
$$

Here, $k$ is the photon energy, $L$ is the multipolarity of the radiative field, $\kappa=(\ell-j)(2 j+1)$ is the relativistic quantum number, $j$ and $\ell$ are the total and orbital momentum of the electron and $\alpha$ is the fine structure constant. Equation (1) is written per one electron. Relativistic units ( $\left.\hbar=m_{0}=c=1\right)$ are used in equations throughout this text, unless otherwise specified. The reduced matrix element $Q_{\Lambda L}(\kappa)$ has the form:

$$
\begin{aligned}
Q_{\Lambda L}(\kappa) & =\left[(2 \bar{\ell}+1)\left(2 \ell_{i}+1\right) /(2 \Lambda+1)\right]^{1 / 2} C_{\bar{\ell}_{0} \ell_{i} 0}^{\Lambda 0} \mathcal{A}\left(\begin{array}{ccc}
\bar{\ell} & 1 / 2 & j \\
\ell_{i} & 1 / 2 & j_{i} \\
\Lambda & 1 & L
\end{array}\right) R_{1 \Lambda} \\
& +\left[(2 \ell+1)\left(2 \bar{\ell}_{i}+1\right) /(2 \Lambda+1)\right]^{1 / 2} C_{\ell 0 \bar{\ell}_{i} 0}^{\Lambda 0} \mathcal{A}\left(\begin{array}{ccc}
\ell & 1 / 2 & j \\
\bar{\ell}_{i} & 1 / 2 & j_{i} \\
\Lambda & 1 & L
\end{array}\right) R_{2 \Lambda}
\end{aligned}
$$

where $\bar{\ell}=2 j-\ell, C_{\ell_{1} 0 \ell_{2} 0}^{\Lambda 0}$ is the Clebsch-Gordan coefficient and $\mathcal{A}\left(\begin{array}{ccc}\ell_{1} & 1 / 2 & j_{1} \\ \ell_{2} & 1 / 2 & j_{2} \\ \Lambda & 1 & L\end{array}\right)$ is the recoupling coefficient for four angular momenta. Radial integrals $R_{1 \Lambda}$ and $R_{2 \Lambda}$ are given by:

$$
\begin{aligned}
R_{1 \Lambda} & =\int_{0}^{\infty} G_{i}(r) F(r) j_{\Lambda}(k r) d r \\
R_{2 \Lambda} & =\int_{0}^{\infty} G(r) F_{i}(r) j_{\Lambda}(k r) d r
\end{aligned}
$$

where $G(r)$ and $F(r)$ are the large and small components of the Dirac electron wave function multiplied by $r$ and $j_{\Lambda}(k r)$ is the spherical Bessel function of the $\Lambda$-th order. The subscript $i \equiv n_{i} \ell_{i} j_{i} \equiv n_{i} \kappa_{i}$ relates to the bound electron state, while designations with no subscript relate to the continuum state. The electron wave functions are calculated in the framework of the DF method where the exchange electron interaction is included exactly both between bound electrons and between bound and free electrons [18]. The bound and continuum wave functions are calculated in the self-consistent fields (SCF) of the corresponding ions with $N+1$ and $N$ electrons, respectively.

The partial RRCS $\sigma_{\mathrm{rr}}^{(i)}$ for a recombining ion $\mathrm{W}^{q+}$, as an example, can be expressed in terms of PCS $\sigma_{\mathrm{ph}}^{(i)}$ for the associated recombined ion $\mathrm{W}^{(q-1)+}$, which makes up as the recombining ion with one additional electron in the $\mathrm{i}$-th subshell with quantum numbers $n_{i}, \ell_{i}$ and $j_{i}$ :

$$
W^{(q-1)+}=W^{q+}+\left(n_{i} \ell_{i} j_{i}\right)
$$


We use the relativistic relationship between PCS and RRCS, which may be written as [23]:

$$
\sigma_{\mathrm{rr}}^{(i)}=\frac{k^{2}}{2 m_{0} c^{2} E_{\mathrm{k}}+E_{\mathrm{k}}^{2}} q^{(i)} \sigma_{\mathrm{ph}}^{(i)}
$$

where $E_{k}$ is the kinetic electron energy and $q^{(i)}$ is the number of vacancies in the $\mathrm{i}$-th subshell prior to recombination.

The relativistic RR rates $\alpha_{\text {rel }}^{(i)}(T)$ can be found using the thermal average over the relativistic RRCS/PCS provided the continuum electron velocity is described by the relativistic Maxwell-Jüttner distribution. The associated distribution function $f(E)$ normalized to unity is written as follows [24]

$$
f(E) d E=\frac{E\left(E^{2}-1\right)^{1 / 2}}{\theta \exp (1 / \theta) K_{2}(1 / \theta)} \times \exp [-(E-1) / \theta] d E
$$

Here, $E$ is the total electron energy in units of $m_{0} c^{2}$, including the rest energy, $\theta=k_{\beta} T / m_{0} c^{2}$ is the characteristic dimensionless temperature, $k_{\beta}$ is the Boltzmann constant and $T$ is the temperature. The modified Bessel function of the second order is denoted by $K_{2}$.

Taking account of the relativistic distribution (Equation (6)) along with the relativistic relationship (Equation (5)), the expression for the relativistic RR rates takes the factorized form:

$$
\alpha_{\mathrm{rel}}^{(i)}(T)=<v \sigma_{\mathrm{rr}}^{(i)}>=F_{\mathrm{rel}}(\theta) \cdot \alpha^{(i)}(T)
$$

Here, $v=(p / E) c$ is the electron velocity with the momentum $p=\sqrt{E^{2}-1}$. The factor $\alpha^{(i)}(T)$ is the usual RR rate with the non-relativistic Maxwell-Boltzmann distribution, which can be written as:

$$
\alpha^{(i)}(T)=(2 / \pi)^{1 / 2} c^{-2}\left(m_{0} k_{\beta} T\right)^{-3 / 2} q^{(i)} \int_{\varepsilon_{i}}^{\infty} k^{2} \sigma_{\mathrm{ph}}^{(i)}(k) e^{\left(\varepsilon_{i}-k\right) /\left(k_{\beta} T\right)} d k
$$

where $\varepsilon_{i}$ is the ionization threshold energy of the i-th subshell. According to Equation (7), to obtain the relativistic RR rates, $\alpha^{(i)}(T)$, involving relativistic values of $\sigma_{\mathrm{ph}}^{(i)}(k)$, should be multiplied by the relativistic factor $F_{\text {rel }}(\theta)$, which is written as:

$$
F_{\text {rel }}(\theta)=\sqrt{\frac{\pi}{2} \theta} / K_{2}(1 / \theta) \exp (1 / \theta)
$$

This is just the factor that comes from the relativistic Maxwell-Jüttner distribution with allowance made for relativistic relationship between PCS and RRCS. The factor has been obtained for the first time by us $[6,7,25]$. It has been taken into consideration in all our calculations of RR/RPL rates.

Similarly, the expression for relativistic RPL rates can be found as:

$$
\gamma_{\mathrm{rel}}^{(i)}(T)=\left\langle v k \sigma_{\mathrm{rr}}^{(i)}\right\rangle=F_{\mathrm{rel}}(\theta) \cdot \gamma^{(i)}(T)
$$

Here, $\gamma^{(i)}(T)$ is the RPL rate obtained using the non-relativistic Maxwell-Boltzmann electron distribution:

$$
\gamma^{(i)}(T)=(2 / \pi)^{1 / 2} c^{-2}\left(m_{0} k_{\beta} T\right)^{-3 / 2} q^{(i)} \int_{\varepsilon_{i}}^{\infty} k^{3} \sigma_{\mathrm{ph}}^{(i)}(k) e^{\left(\varepsilon_{i}-k\right) /\left(k_{\beta} T\right)} d k
$$

Note once again that Equations (8) and (11) have to involve relativistic values of PCS. Numerical methods used for calculations of integrals in Equations (8) and (11) are described at length in [7]. 


\subsection{Results and Discussion}

The influence of relativistic, non-dipole and exchange effects on PCS, RRCS and RR/RPL rates was considered in [7,25]. It is significant that relativistic and non-dipole effects are of importance for highly-charged ions and at high energies. For example, as is evident from Figure 1, where the factor $F_{\text {rel }}(\theta)$ is displayed versus temperature, the relativistic Maxwell-Jüttner distribution decreases $\mathrm{RR} / \mathrm{RPL}$ rates considerably at a high temperature as compared with the commonly-used non-relativistic Maxwell-Boltzmann distribution. For example, the decreasing is $\sim 25 \%$ at the highest temperature in our calculations $T=10^{9} \mathrm{~K} \approx 86 \mathrm{keV}$.

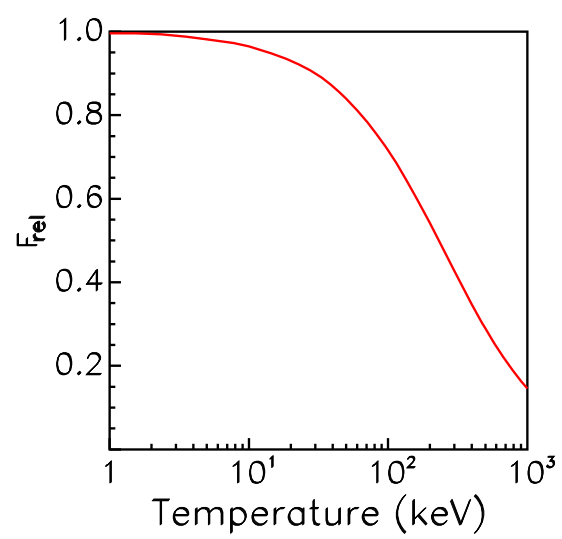

Figure 1. The relativistic factor $F_{\text {rel }}$ for radiative recombination (RR) rates and radiated power loss (RPL) rates.

On the contrary, the exact consideration of the electron exchange is of importance in the RR and photoionization calculations for low-charged ions, especially at low energies. Partial RRCS for recombination of $\mathrm{W}^{6+}$ with an electron captured in the $5 d_{3 / 2}, 5 f_{5 / 2}$ and $6 p_{1 / 2}$ states calculated by the DF method having regard to the exact exchange (red curves) and by the DS method having regard to the approximate exchange according to Slater (blue curves) [16] are presented in Figure 2. As is seen, there is a significant difference between the two calculations especially at low electron energies and in the vicinity of the Cooper minimum. The exact consideration of the electron exchange may change partial RRCS and PCS by several times at electron energies $E_{k} \lesssim 1,000 \mathrm{eV}$ and up by $\sim 70 \%$ at high electron energies.

Calculations were performed for the most stable tungsten ions. For each ion, the electron configuration with the lowest total energy was found by the DF method taking into account the Breit magnetic interaction between electrons. It should be noted that all ion configurations obtained by us coincide with those presented in compilation [26], where the experimentally-derived energy levels are used. The adopted electron configuration for 54 ions considered in the framework of CRP are listed in Table 1 along with the values of total energies. For these ions, partial and total RRCS were calculated for 46 values of the electron energy $E_{\mathrm{k}}$. The energies are logarithmic over the range $4 \mathrm{eV} \leq E_{\mathrm{k}} \lesssim 80 \mathrm{keV}$. In addition, three values near the threshold, $E_{\mathrm{k}}=1,2,3 \mathrm{eV}$, are included. Partial $\alpha_{\mathrm{rel}}^{(i)}(T)$ and total $\alpha_{\text {tot }}(T)$ RR rates, as well as partial $\gamma_{\text {rel }}^{(i)}(T)$ and total $\gamma_{\text {tot }}(T)$ RPL rates were calculated for eleven values of temperature in the range from $10^{4} \mathrm{~K}-10^{9} \mathrm{~K}$, i.e., from $0.86 \mathrm{eV}-86 \mathrm{keV}$. 


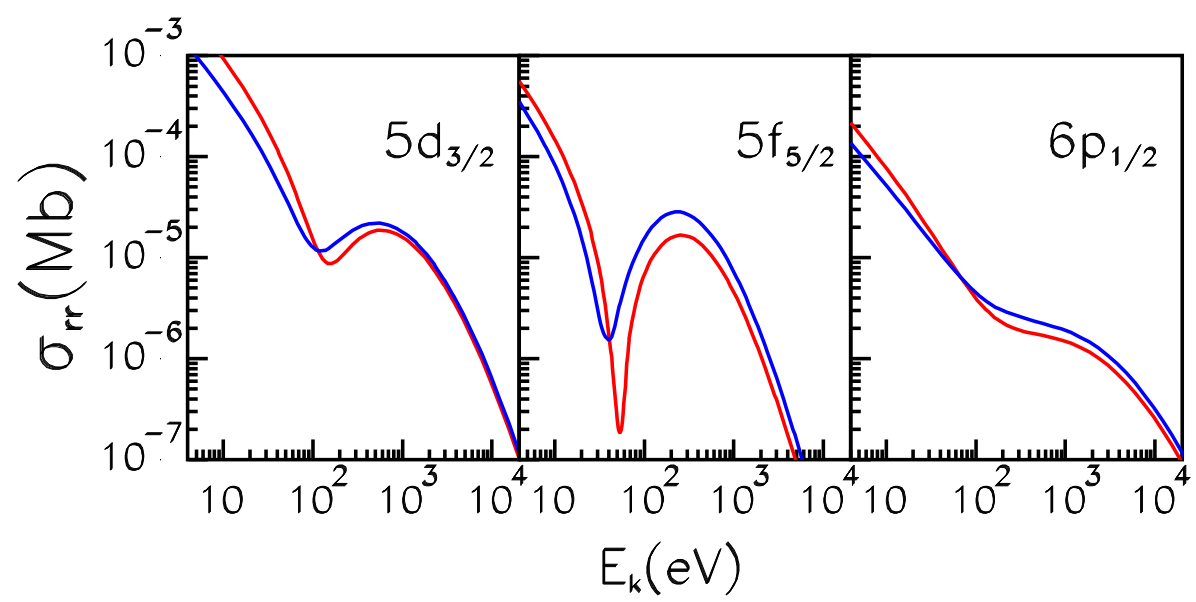

Figure 2. Partial RR cross-sections (RRCS) for $\mathrm{RR}$ of $\mathrm{W}^{6+}$ with an electron captured in the $5 d_{3 / 2}, 5 f_{5 / 2}$ and $6 p_{1 / 2}$ states. Red, Dirac-Fock (DF) calculation with regard to the exact electron exchange; blue, Dirac-Slater (DS) calculation with approximate consideration for the exchange.

Table 1. Electron configurations adopted for tungsten ions along with total energies calculated by the DF method taking into account the Breit magnetic interaction between electrons.

\begin{tabular}{|c|c|c|c|c|c|}
\hline Ion & Configuration & $-E_{\text {tot }}(\mathrm{eV})$ & Ion & Configuration & $-E_{\text {tot }}(\mathrm{eV})$ \\
\hline $\mathrm{W}^{6+}$ & {$[\mathrm{Xe}] 4 f_{5 / 2}^{6} 4 f_{7 / 2}^{8}$} & $438,971.5$ & $\mathrm{~W}^{42+}$ & {$[\mathrm{Ar}] 3 d_{3 / 2}^{4} 3 d_{5 / 2}^{6} 4 s^{2} 4 p_{1 / 2}^{2}$} & $407,964.5$ \\
\hline $\mathrm{W}^{14+}$ & {$[\mathrm{Kr}] 4 d_{3 / 2}^{4} 4 d_{5 / 2}^{6} 4 f_{5 / 2}^{6} 4 f_{7 / 2}^{6} 5 s_{1 / 2}^{2}$} & $437,397.8$ & $\mathrm{~W}^{43+}$ & {$[\mathrm{Ar}] 3 d_{3 / 2}^{4} 3 d_{5 / 2}^{6} 4 s^{2} 4 p_{1 / 2}^{1}$} & $405,819.8$ \\
\hline $\mathrm{W}^{15+}$ & {$[\mathrm{Kr}] 4 d_{3 / 2}^{4} 4 d_{5 / 2}^{6} 4 f_{5 / 2}^{6} 4 f_{7 / 2}^{5} 5 s_{1 / 2}^{2}$} & $437,072.6$ & $\mathrm{~W}^{44+}$ & {$[\mathrm{Ar}] 3 d_{3 / 2}^{4} 3 d_{5 / 2}^{6} 4 s^{2}$} & $403,613.8$ \\
\hline $\mathrm{W}^{16+}$ & {$[\mathrm{Kr}] 4 d_{3 / 2}^{4} 4 d_{5 / 2}^{6} 4 f_{5 / 2}^{6} 4 f_{7 / 2}^{5} 5 s_{1 / 2}^{1}$} & $436,712.7$ & $\mathrm{~W}^{45+}$ & {$[\mathrm{Ar}] 3 d_{3 / 2}^{4} 3 d_{5 / 2}^{6} 4 s^{1}$} & $401,260.9$ \\
\hline $\mathrm{W}^{17+}$ & {$[\mathrm{Kr}] 4 d_{3 / 2}^{4} 4 d_{5 / 2}^{6} 4 f_{5 / 2}^{6} 4 f_{7 / 2}^{5}$} & $436,327.5$ & $\mathrm{~W}^{47+}$ & {$[\mathrm{Ar}] 3 d_{3 / 2}^{4} 3 d_{5 / 2}^{5}$} & $394,795.1$ \\
\hline $\mathrm{W}^{18+}$ & {$[\mathrm{Kr}] 4 d_{3 / 2}^{4} 4 d_{5 / 2}^{6} 4 f_{5 / 2}^{6} 4 f_{7 / 2}^{4}$} & $435,907.9$ & $\mathrm{~W}^{48+}$ & {$[\mathrm{Ar}] 3 d_{3 / 2}^{4} 3 d_{5 / 2}^{4}$} & $390,616.1$ \\
\hline $\mathrm{W}^{19+}$ & {$[\mathrm{Kr}] 4 d_{3 / 2}^{4} 4 d_{5 / 2}^{6} 4 f_{5 / 2}^{6} 4 f_{7 / 2}^{3}$} & $435,448.6$ & $\mathrm{~W}^{49+}$ & {$[\mathrm{Ar}] 3 d_{3 / 2}^{4} 3 d_{5 / 2}^{3}$} & $386,307.9$ \\
\hline $\mathrm{W}^{20+}$ & {$[\mathrm{Kr}] 4 d_{3 / 2}^{4} 4 d_{5 / 2}^{6} 4 f_{5 / 2}^{6} 4 f_{7 / 2}^{2}$} & $434,948.3$ & $\mathrm{~W}^{50+}$ & {$[\mathrm{Ar}] 3 d_{3 / 2}^{4} 3 d_{5 / 2}^{2}$} & $381,869.1$ \\
\hline $\mathrm{W}^{21+}$ & {$[\mathrm{Kr}] 4 d_{3 / 2}^{4} 4 d_{5 / 2}^{6} 4 f_{5 / 2}^{6} 4 f_{7 / 2}^{1}$} & $434,405.8$ & $\mathrm{~W}^{51+}$ & {$[\mathrm{Ar}] 3 d_{3 / 2}^{4} 3 d_{5 / 2}^{1}$} & $377,298.0$ \\
\hline $\mathrm{W}^{22+}$ & {$[\mathrm{Kr}] 4 d_{3 / 2}^{4} 4 d_{5 / 2}^{6} 4 f_{5 / 2}^{6}$} & $433,819.9$ & $\mathrm{~W}^{52+}$ & {$[\mathrm{Ar}] 3 d_{3 / 2}^{4}$} & $372,593.2$ \\
\hline $\mathrm{W}^{23+}$ & {$[\mathrm{Kr}] 4 d_{3 / 2}^{4} 4 d_{5 / 2}^{6} 4 f_{5 / 2}^{5}$} & $433,181.3$ & $\mathrm{~W}^{53+}$ & {$[\mathrm{Ar}] 3 d_{3 / 2}^{3}$} & $367,673.9$ \\
\hline $\mathrm{W}^{24+}$ & {$[\mathrm{Kr}] 4 d_{3 / 2}^{4} 4 d_{5 / 2}^{6} 4 f_{5 / 2}^{4}$} & $432,496.8$ & $\mathrm{~W}^{54+}$ & {$[\mathrm{Ar}] 3 d_{3 / 2}^{2}$} & $362,615.4$ \\
\hline $\mathrm{W}^{25+}$ & {$[\mathrm{Kr}] 4 d_{3 / 2}^{4} 4 d_{5 / 2}^{6} 4 f_{5 / 2}^{3}$} & $431,765.3$ & $\mathrm{~W}^{55+}$ & {$[\mathrm{Ar}] 3 d_{3 / 2}^{1}$} & $357,416.2$ \\
\hline $\mathrm{W}^{26+}$ & {$[\mathrm{Kr}] 4 d_{3 / 2}^{4} 4 d_{5 / 2}^{6} 4 f_{5 / 2}^{2}$} & $430,985.6$ & $\mathrm{~W}^{57+}$ & {$[\mathrm{Ne}] 3 s^{2} 3 p_{1 / 2}^{2} 3 p_{3 / 2}^{3}$} & $346,348.4$ \\
\hline $\mathrm{W}^{27+}$ & {$[\mathrm{Kr}] 4 d_{3 / 2}^{4} 4 d_{5 / 2}^{6} 4 f_{5 / 2}^{1}$} & $430,156.8$ & $\mathrm{~W}^{58+}$ & {$[\mathrm{Ne}] 3 s^{2} 3 p_{1 / 2}^{2} 3 p_{3 / 2}^{2}$} & $340,497.7$ \\
\hline$W^{29+}$ & {$[\mathrm{Kr}] 4 d_{3 / 2}^{4} 4 d_{5 / 2}^{5}$} & $428,149.4$ & $\mathrm{~W}^{59+}$ & {$[\mathrm{Ne}] 3 s^{2} 3 p_{1 / 2}^{2} 3 p_{3 / 2}^{1}$} & $334,522.0$ \\
\hline $\mathrm{W}^{30+}$ & {$[\mathrm{Kr}] 4 d_{3 / 2}^{4} 4 d_{5 / 2}^{4}$} & $426,971.7$ & $\mathrm{~W}^{60+}$ & {$[\mathrm{Ne}] 3 s^{2} 3 p_{1 / 2}^{2}$} & $328,420.1$ \\
\hline
\end{tabular}


Table 1. Cont.

\begin{tabular}{cccccc}
\hline Ion & Configuration & $-\boldsymbol{E}_{\text {tot }}(\mathbf{e V})$ & Ion & Configuration & $-\boldsymbol{E}_{\text {tot }}(\mathbf{e V})$ \\
\hline $\mathrm{W}^{31+}$ & {$[\mathrm{Kr}] 4 d_{3 / 2}^{4} 4 d_{5 / 2}^{3}$} & $425,744.0$ & $\mathrm{~W}^{61+}$ & {$[\mathrm{Ne}] 3 s^{2} 3 p_{1 / 2}^{1}$} & $321,835.9$ \\
$\mathrm{~W}^{32+}$ & {$[\mathrm{Kr}] 4 d_{3 / 2}^{4} 4 d_{5 / 2}^{2}$} & $424,465.6$ & $\mathrm{~W}^{62+}$ & {$[\mathrm{Ne}] 3 s^{2}$} & $315,110.6$ \\
$\mathrm{~W}^{33+}$ & {$[\mathrm{Kr}] 4 d_{3 / 2}^{4} 4 d_{5 / 2}^{1}$} & $423,135.8$ & $\mathrm{~W}^{63+}$ & {$[\mathrm{Ne}] 3 s^{1}$} & $308,108.4$ \\
$\mathrm{~W}^{34+}$ & {$[\mathrm{Kr}] 4 d_{3 / 2}^{4}$} & $421,754.1$ & $\mathrm{~W}^{65+}$ & {$[\mathrm{He}] 2 s^{2} 2 p_{1 / 2}^{2} 2 p_{3 / 2}^{3}$} & $285,369.8$ \\
$\mathrm{~W}^{35+}$ & {$[\mathrm{Kr}] 4 d_{3 / 2}^{3}$} & $420,299.3$ & $\mathrm{~W}^{66+}$ & {$[\mathrm{He}] 2 s^{2} 2 p_{1 / 2}^{2} 2 p_{3 / 2}^{2}$} & $269,426.1$ \\
$\mathrm{~W}^{36+}$ & {$[\mathrm{Kr}] 4 d_{3 / 2}^{2}$} & $418,790.9$ & $\mathrm{~W}^{67+}$ & {$[\mathrm{He}] 2 s^{2} 2 p_{1 / 2}^{2} 2 p_{3 / 2}^{1}$} & $253,138.4$ \\
$\mathrm{~W}^{37+}$ & {$[\mathrm{Kr}] 4 d_{3 / 2}^{1}$} & $417,228.1$ & $\mathrm{~W}^{68+}$ & {$[\mathrm{He}] 2 s^{2} 2 p_{1 / 2}^{2}$} & $236,503.6$ \\
$\mathrm{~W}^{39+}$ & {$[\mathrm{Ar}] 3 d_{3 / 2}^{4} 3 d_{5 / 2}^{6} 4 s^{2} 4 p_{1 / 2}^{2} 4 p_{3 / 2}^{3}$} & $413,781.3$ & $\mathrm{~W}^{69+}$ & {$[\mathrm{He}] 2 s^{2} 2 p_{1 / 2}^{1}$} & $218,086.5$ \\
$\mathrm{~W}^{40+}$ & {$[\mathrm{Ar}] 3 d_{3 / 2}^{4} 3 d_{5 / 2}^{6} 4 s^{2} 4 p_{1 / 2}^{2} 4 p_{3 / 2}^{2}$} & $411,897.7$ & $\mathrm{~W}^{70+}$ & {$[\mathrm{He}] 2 s^{2}$} & $199,257.1$ \\
$\mathrm{~W}^{41+}$ & {$[\mathrm{Ar}] 3 d_{3 / 2}^{4} 3 d_{5 / 2}^{6} 4 s^{2} 4 p_{1 / 2}^{2} 4 p_{3 / 2}^{1}$} & $409,958.8$ & $\mathrm{~W}^{71+}$ & {$[\mathrm{He}] 2 s^{1}$} & $179,889.0$ \\
\hline
\end{tabular}

To have a chance of getting partial cross-sections at any energies and presenting a great body of data in compact form, PCS for all electron states with principal quantum numbers $n \leq 10$ and orbital momenta $\ell \leq 4$ were fitted by the following analytical expression [27]:

$$
\sigma_{\mathrm{ph}}^{(n \ell j)}(k)=\sigma_{0}\left\{\left[\left(k / k_{0}-1\right)^{2}+y_{w}^{2}\right]\left(k / k_{0}\right)^{0.5 p-\ell-5.5}\left[1+\sqrt{k /\left(k_{0} y_{\mathrm{a}}\right)}\right]^{-p}\right\}
$$

where $\sigma_{0}, k_{0}, y_{\mathrm{w}}, p$ and $y_{\mathrm{a}}$ are fit parameters. With Equation (12), the fit parameters were found by minimizing the mean-square deviation of fitted PCS from calculated values with the simplex search method. The fitting was performed in the following range of the photon energy:

$$
\varepsilon_{n \ell j}+1 \mathrm{eV} \leq k \leq k_{\max }
$$

where $\varepsilon_{n \ell j} \equiv \varepsilon_{i}$ is the ionization threshold energy. The maximum fitting energy $k_{\max }$ is determined by decreasing PCS $\sigma_{\mathrm{ph}}^{(n \ell j)}\left(k_{\max }\right)$ as compared with its maximum by five/six orders of magnitude. Usually, $k_{\max }$ is of the order of a few hundred of $\varepsilon_{n \ell j}$ for the $s, p$ and $d$ states and of a few tens of $\varepsilon_{n \ell j}$ for the $f$ and $g$ states. Consequently, the fit parameters and Equation (12) allow one to obtain PCS at any value of $k \leq k_{\max }$. The associated value of RRCS is readily obtainable using Equation (5).

Calculations of partial PCS are performed with a numerical precision $0.1 \%$. However, the accuracy may be changed in the course of fitting. Therefore, the real root-mean-square (RMS) error $\delta_{\mathrm{av}}$ was found for each a state as follows:

$$
\delta_{\mathrm{av}}=\sqrt{\frac{1}{M} \sum_{m=1}^{M}\left[\frac{\sigma_{\mathrm{phc}}^{(n \ell j)}\left(k_{m}\right)-\sigma_{\mathrm{phf}}^{(n \ell j)}\left(k_{m}\right)}{\sigma_{\mathrm{phc}}^{(n \ell j)}\left(k_{m}\right)}\right]^{2}} \cdot 100 \%
$$

where $M$ is the number of the energy points involved in the fitting and $\sigma_{\mathrm{phc}}^{(n \ell j)}$ and $\sigma_{\mathrm{phf}}^{(n \ell j)}$ are calculated and fitted values of PCS, respectively. As a rule, the error is $\delta_{\text {av }} \lesssim 2 \%$. However, for comparatively low-charged ions, the RMS error may be larger. For example, for the $n f$ shells with $n \geq 5$, as well as for the $n s$ and $n p$ shells with $n \geq 7$ of $\mathrm{W}^{14+}$, the maximal error reaches $\sim 7 \%$. The PCS fit parameters are presented for recombined ions along with associated ionization threshold energies $\varepsilon_{n \ell j}$, maximum fitting energies $k_{\max }$ and RMS errors $\delta_{\mathrm{av}}$ (see [5,7-10]). 
Total RRCS $\sigma_{\text {tot }}\left(E_{\mathrm{k}}\right)$ are determined by summation of partial values over all electron states beginning from the lowest open shell up to shells with the principal quantum number $n=20$ as follows [5,7]:

$$
\sigma_{\text {tot }}\left(E_{\mathrm{k}}\right)=\sum_{n=n_{\min }}^{20} \sum_{\kappa=\mp 1}^{-n} \sigma_{\mathrm{rr}}^{(n \ell j \equiv n \kappa)}\left(E_{\mathrm{k}}\right)
$$

where $n_{\min }$ along with a corresponding value of $\kappa$ refers to the ground state. To obtain total RR/RPL rates, summations over electron states are performed in the same manner.

A part of our calculations refers to the comparatively low-charged tungsten ions with charges $6 \leq q \lesssim 20$. A peculiarity of the ions is that RRCS, PCS as well as RR/RPL rates may be bent or even nonmonotonic functions of the energy. Such ions are more difficult for calculations and especially for fitting. Figure 3 demonstrates total RRCS (3a), RR rates (3b) and RPL rates (3c) for eight representative tungsten ions in the charge range $6 \leq q \leq 57$. As is seen, curves $\sigma_{\text {tot }}\left(E_{\mathrm{k}}\right)$ and $\alpha_{\text {tot }}(T)$ for ions with $q \lesssim 20$ have noticeable bends at energies $\sim 100-300 \mathrm{eV}$. This tendency brings into existence the minimum and maximum in $\sigma_{\text {tot }}\left(E_{k}\right)$, explicit bends in $\alpha_{\text {tot }}(T)$ and into the oscillating curve $\gamma_{\text {tot }}(T)$ for the low-charged tungsten ion $\mathrm{W}^{6+}$ (rose curves). The $E_{k}$-dependence of $\sigma_{\text {tot }}$ and the $T$-dependence of $\alpha_{\text {tot }}$ for ions with $q \gtrsim 20$ become smooth curves. As is evident from Figure 3c, the behavior of total RPL rates is nonmonotonic for ions $\mathrm{W}^{6+}-\mathrm{W}^{17+}$ and has noticeable bends up to $\mathrm{W}^{45+}$. Only for $\mathrm{W}^{57+}$, the curve $\gamma_{\text {tot }}(T)$ becomes smooth.
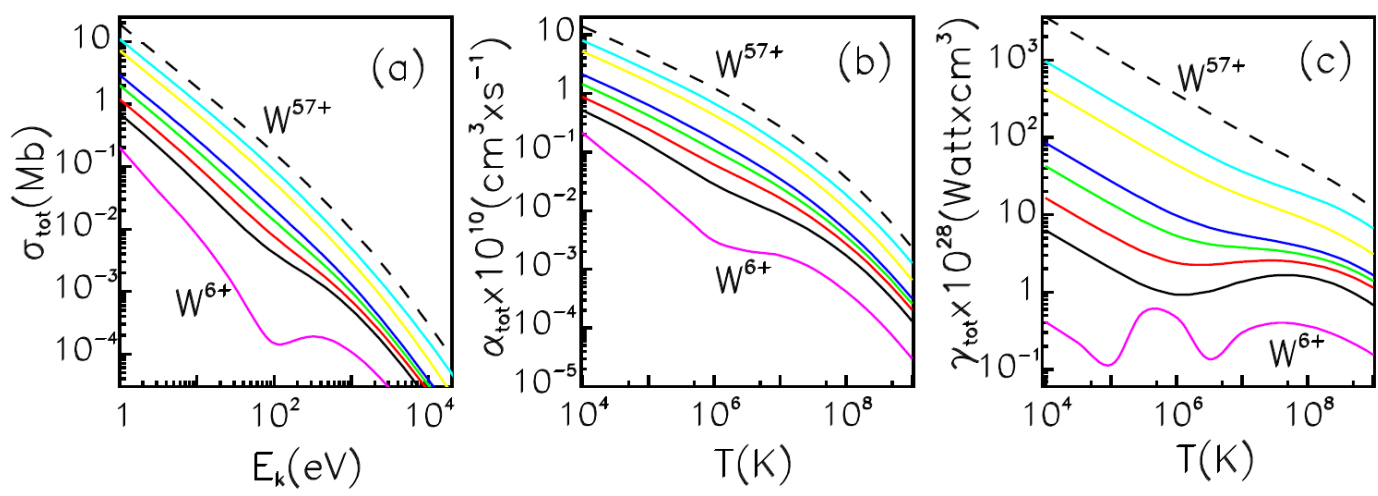

Figure 3. Total RRCS (a), RR rates (b) and RPL rates (c) for representative tungsten ions. Rose, $\mathrm{W}^{6+}$; black solid, $\mathrm{W}^{14+}$; red, $\mathrm{W}^{17+}$; green, $\mathrm{W}^{20+}$; blue, $\mathrm{W}^{23+}$; yellow, $\mathrm{W}^{35+}$; light blue, $\mathrm{W}^{45+}$; black dashed $\mathrm{W}^{57+}$.

It is self-evident that such a structure of total cross-sections and rates for low-charged ions is caused by the behavior of the associated partial cross-sections and rates. Displayed in Figure 4 are partial RRCS (4a), RR rates (4b) and RPL rates (4c) for states contributing significantly to the relevant total values for $\mathrm{W}^{6+}$. The oscillating and bent $E_{\mathrm{k}} / T$-dependence of the $5 d, 5 f, 6 s, 6 p$ and other higher states manifests itself in total RRCS and RR/RPL rates. Total RRCS are found by summation up to states with $n=20$ (see Equation (15)). For low-charged ions, like $\mathrm{W}^{6+}$, all $n d$ and $n f$ states with, at least, $n \lesssim 15$ have a bent or oscillating structure. Certainly, the contributions of higher states are less. For example, contributions of states with $n=6$ are $\sim 3-4$-times less than of states with $n=5$, and contributions of states with $n=7$ are $\sim 2$-times less than of states with $n=6$, etc. However, these states contribute 
noticeably. It should be noted that increasing the fitting error $\delta_{\mathrm{av}}$ mentioned above is just due to such behavior of partial PCS and RRCS.
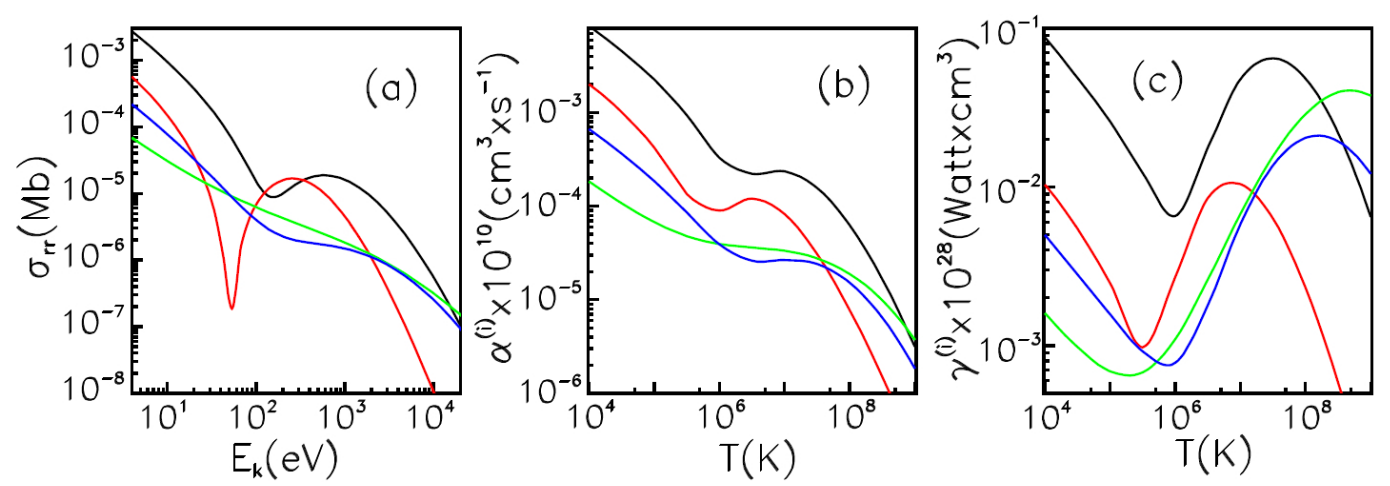

Figure 4. Partial RRCS (a), RR rates (b) and RPL rates (c) for the recombination of the $\mathrm{W}^{6+}$ ion with an electron captured in various states. Black, the $5 d_{3 / 2}$; red, the $5 f_{5 / 2}$; green, the $6 s_{1 / 2}$; blue, the $6 p_{1 / 2}$.

We would like to emphasize that RR and RPL rates for the ion $\mathrm{W}^{6+}$ were first obtained in this work. Partial and total values of the rates are presented in Tables A1 and A2 in Appendix.

Although total RR rates were computed at eleven values of temperature, an analytical expression is convenient to use in fusion studies. Therefore, total RR rates for tungsten ions under consideration were fitted by the following expression [8,28]:

$$
\alpha_{\text {tot }}(T)=a\left[\sqrt{T / T_{0}}\left(1+\sqrt{T / T_{0}}\right)^{1.5-b}\left(1+\sqrt{T / T_{1}}\right)^{2.5+b}\right]^{-1}
$$

where $a, b, T_{0}$ and $T_{1}$ are fit parameters. The temperature range of the fitting is from $10^{4} \mathrm{~K}-10^{9} \mathrm{~K}$. The RMS error was calculated using the expression that is analogous to Equation (14) with $M=11$. Usually, the RMS error is $\lesssim 1.5 \%$. Note once more that the fitting becomes less accurate when the ion charge decreases. The fit parameters for $\alpha_{\text {tot }}(T)$ together with the associated RMS error $\delta_{\text {av }}$ are presented for all tungsten ions in [8-11].

\section{Polarization Radiative Recombination Effect}

\subsection{Model Used in Calculations}

To obtain more accurate values of RRCS, we estimated the impact on RRCS of the core electron polarization following the RR process for highly-charged ions. It has been revealed previously (see [29] and the references therein) that the standard RR probability may be enhanced due to virtual excitations (polarization) of the ion core electrons by the Coulomb field of an incident electron. In the PRR process, the photon is emitted not by the incident electron as in the RR process, but by core electrons. The RR and PRR amplitudes are shown in Figure 5a,b, respectively. 
(a)

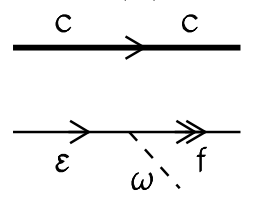

(b)

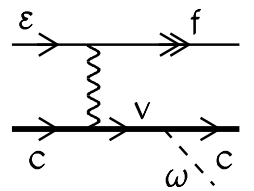

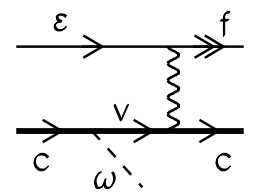

Figure 5. Feynman diagrams for amplitudes of the RR (a) and PRR (b) processes.

Here, thin lines describe the transition of the incident electron with the energy $\varepsilon$ into the bound $f$-state. Thick lines "c" relate to the target electrons and "v" to their virtual states. Dashed lines denote the emitted photon with the energy $\omega$. The Coulomb interaction is indicated with wave lines.

Since the initial continuum electron states $\varepsilon$ and final bound states $f$ in the RR and PRR amplitudes are identical, there is quantum interference between them. At energies of dielectronic resonances, PRR is indistinguishable from an interfering part of dielectronic recombination (DR), leading to the final state with a single excited electron. Quantum interference between RR and DR for highly-charged ions was first considered in [30]. In energy regions free from dielectronic resonances, PRR is the dominant process, where its main effect is the enhancement of the RR background [31,32]. Such non-resonance photon energy ranges for polarization of the $n \ell j$ shells may be written as:

$$
\varepsilon_{n s} \lesssim k \lesssim \varepsilon_{(n-1) \ell_{\max } j_{\max }}
$$

where $\varepsilon_{n s}$ is the ionization energy of the $n s$ shell and $\varepsilon_{(n-1)} \ell_{\max } j_{\max }$ is the ionization energy of the most outer subshell with the principal quantum number $n-1$. Such energy intervals may be rather wide, because ionization potentials are well separated for highly-charged ions. The total cross-section of the $\mathrm{RR}$ and PRR processes for intervals defined by Equation (17) is written as:

$$
\sigma_{(\mathrm{tot})}=\sigma_{\mathrm{rr}}+\sigma_{\mathrm{int}}+\sigma_{\mathrm{prr}}
$$

where $\sigma_{\mathrm{rr}}$ is the standard RRCS, $\sigma_{\mathrm{prr}}$ is the PRR cross-section and $\sigma_{\text {int }}$ is the interference term. The term $\sigma_{\text {prr }}$ was shown in $[29,31,32]$ to be much less than $\sigma_{\text {rr }}$, i.e., $\sigma_{\text {prr }} / \sigma_{\text {rr }} \ll 1$. Therefore, it is the interference term $\sigma_{\text {int }}$, which is responsible for the RRCS enhancement. The contribution to the interference term comes from all virtual electron excitations, including excitations into the continuum. Therefore, the enhancement factor for RRCS due to PRR may be written as:

$$
F_{n}=\frac{\sigma_{(\text {tot })}}{\sigma_{\text {rr }}} \approx 1+\frac{\sigma_{\text {int }}}{\sigma_{\text {rr }}}
$$

where the subscript $n$ denotes the principal quantum number of the polarized shell.

We used the "stripping approximation" for an analytical estimation of the enhancement factor $F_{n}$. The "stripping" approximation is based on the assumption that outer electrons with the ionization energy $\varepsilon_{\text {out }}<k$ are considered as quasi-free. As a result, the enhancement factor is given by the expression [31]:

$$
F_{n} \approx 1+\frac{2 N_{\text {out }}\left(r_{0}\right)}{k^{2} r_{0}^{3}}
$$


where $N_{\text {out }}$ is the number of the outer-shell electrons with the principal quantum number $n$ within the sphere of radius $r_{0}$, which may be written in the relativistic case as:

$$
N_{\text {out }}\left(r_{0}\right)=\int_{0}^{r_{0}} \sum_{\ell j} Q_{n \ell j}\left[G_{n \ell j}^{2}(r)+F_{n \ell j}^{2}(r)\right] d r
$$

Here, quantum numbers $n \ell j$ refer to polarized electrons, and $Q_{n \ell j}$ is the occupation number of the $n \ell j$ shell. According to the quasi-classical theory of radiative transitions [33], the emission of photons with the energy $k$ by an electron is most effective in the turning point $r_{0}$ of the classical trajectory for which the angular electron velocity is close to $k$. Therefore, the distance $r_{0}$ in Equation (21) may be determined as a root of the equation, which is written in atomic units as follows:

$$
E_{k}=-\frac{Z}{r_{0}}+U_{\mathrm{el}}\left(r_{0}\right)+\frac{k^{2} r_{0}^{2}}{2}
$$

where $U_{\mathrm{el}}(r)$ is the electrostatic potential of ion electrons. We used the relativistic DF electron wave functions and the potential $U_{\mathrm{el}}(r)$, because highly-charged ions of tungsten were considered.

To check the validity of this model, we compare enhancement factors $F_{n}$ obtained within the analytical "stripping" approximation by the use of the relativistic DF electron wave functions with the exact non-relativistic Hartree-Fock values obtained in [31] for the Ni-like and Ne-like ions of $\mathrm{Ru}, \mathrm{Cd}$ and Xe. Factors $F_{2}$ and $F_{3}$ for the Ni-like ions are displayed in Figure 6. Here, the RR process with a capture of an electron into the $4 p$ state $\left(4 p_{1 / 2}\right.$ in the relativistic case) is followed by the polarization of ion electrons with $n=3$ in the photon energy range $I_{3 s} \lesssim k \lesssim I_{2 p_{3 / 2}}$ and with $n=2$ in the range $k \gtrsim I_{2 s}$. In the case of $\mathrm{Xe}^{26+}$, the only non-resonant interval is presented.

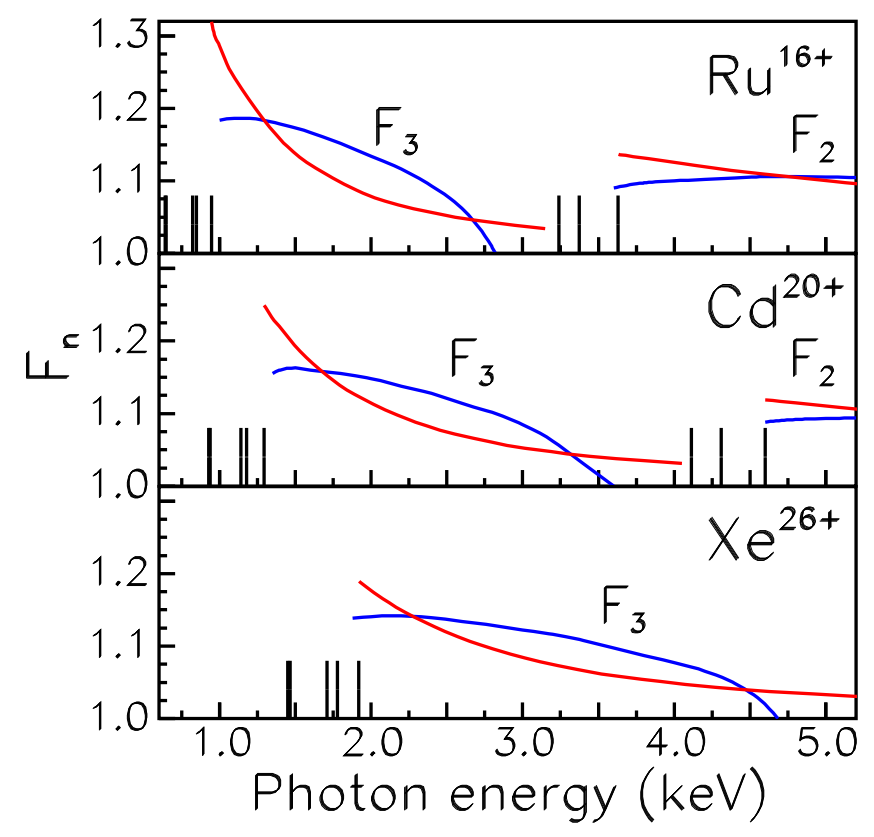

Figure 6. Enhancement factors $F_{2}$ and $F_{3}$ for the capture in the $4 p$ state of the Ni-like ions. Red, present calculations; blue, exact non-relativistic calculations [31]. Vertical lines denote ionization energies obtained in the DF calculations. The lines relate from left to right to the $3 d_{5 / 2}$ and $3 d_{3 / 2}$ states merged together, the $3 p_{3 / 2}, 3 p_{1 / 2}, 3 s$ and the $2 p_{3 / 2}, 2 p_{1 / 2}, 2 s$ states. 
One can see that with exception of the narrow energy ranges close to ionization thresholds, our approximate results (red curves) correlate rather well with exact calculations (blue curves). The agreement becomes better when the ion charge increases. The relative difference between the exact enhancement factor $F_{n}^{(\mathrm{ex})}$ from [31] and our approximate value $F_{n}^{(\text {appr })}$ is written as:

$$
\Delta\left(F_{n}\right)=\left[\left(F_{n}^{(\mathrm{ex})}-F_{n}^{(\mathrm{appr})}\right) / F_{n}^{(\mathrm{ex})}\right] \times 100 \%
$$

The difference $\Delta\left(F_{3}\right)$ does not exceed $6 \%$ for $\mathrm{Ru}^{16+}, 5 \%$ for $\mathrm{Cd}^{20+}$ and $4 \%$ for $\mathrm{Xe}^{26+}$, except for threshold ranges. For the highest-charged ion $\mathrm{Xe}^{26+}, \Delta\left(F_{3}\right)$ equals $\sim 5 \%$ even at the very threshold. The difference $\Delta\left(F_{2}\right)$ is less than $\sim 4 \%$ in the range $k>I_{2 s}$ for ions $\mathrm{Ru}^{16+}$ and $\mathrm{Cd}^{20+}$.

In Figure 7, the similar comparison of factors $F_{2}$ is given for RR of Ne-like ions with an electron captured in the $3 \mathrm{~s}$ shell. As is seen, the difference $\Delta\left(F_{2}\right)$ is small at any electron energy and tends to decrease when the ion charge increases. Maximal $\Delta\left(F_{2}\right)$ changes from $5.5 \%$ for $\mathrm{Ru}^{34+}$ to $3.8 \%$ for $\mathrm{Xe}^{44+}$.

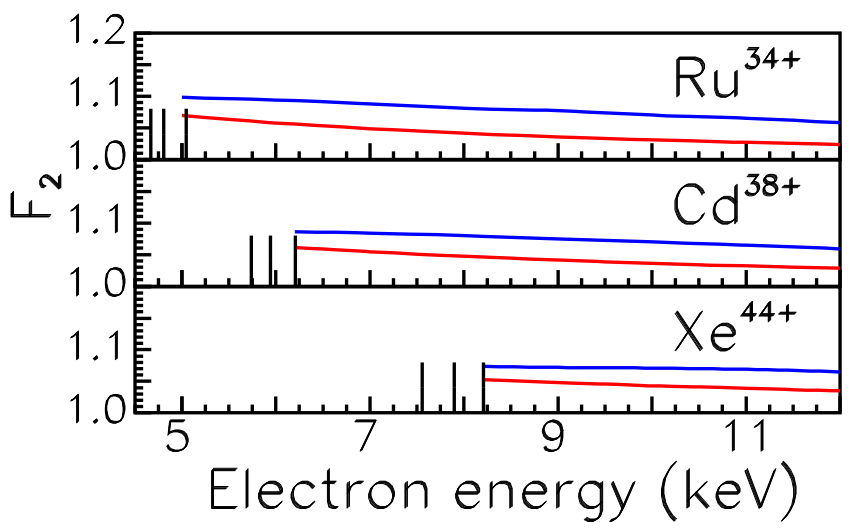

Figure 7. The enhancement factor $F_{2}$ for the capture in the $3 s$ states of the Ne-like ions. Red, present calculations; blue, the exact non-relativistic calculations [31]. Vertical lines denote ionization energies of the $2 p_{3 / 2}, 2 p_{1 / 2}$ and $2 s$ states (from left to right) obtained by the DF method.

\subsection{PRR Effect for Fe XVII}

We assessed the PRR effect for the Ne-like ion Fe XVII to explain the puzzling discrepancy between experimental and theoretical values of EIECS [12]. Measurements of EIECS for dominant X-ray lines from Fe XVII and RR of the beam electrons into the M-shell levels of the source ions were reported in [13]. Absolute values of EIECS $\sigma_{\text {EIE }}$ were determined by normalizing to the measured intensity of the RR peaks, which were, in turn, independently normalized to theoretical RRCS $\sigma_{\text {rr }}$ calculated by the DS method for the $3 s, 3 p$ and $3 d$ states at electron energy $E_{k}=964 \mathrm{eV}$. Experimental values of $\sigma_{\mathrm{EIE}}$ turned out to be lower by $\sim 25 \%$ as compared to all available theoretical EIECS. We assumed that the problem with the determination of absolute values of the measured $\sigma_{\mathrm{EIE}}$ by normalizing the measured intensity of RR peaks to the theoretical $\sigma_{\text {rr }}$ is that only the RR channel is taken into account in the $\sigma_{\text {rr }}$ calculations, while the PRR channel is overlooked.

To account for the PRR channel, we estimated the enhancement factor $F_{2}$ using the "stripping" approximation and the DF method. The resulting value of $F_{2}$, on average, equals 1.22 for RR into 
Table 2. Values of RRCS in barns for RR of the ion Fe XVII with the capture in the $3 \ell j$ electron states calculated by the DF and DS methods at $E_{k}=964 \mathrm{eV}$. $\Delta=\left[\left(\sigma_{\mathrm{DS}}-\sigma_{\mathrm{DF}}\right) / \sigma_{\mathrm{DF}}\right] \times 100 \%$.

\begin{tabular}{cccc}
\hline $\boldsymbol{n} \boldsymbol{\ell} \boldsymbol{j}$ & $\boldsymbol{\sigma}_{\mathrm{DF}}(\mathbf{b a r n})$ & $\boldsymbol{\sigma}_{\mathrm{DS}}(\mathbf{b a r n})$ & $\boldsymbol{\Delta}(\boldsymbol{\%})$ \\
\hline $3 s_{1 / 2}$ & 33.9 & 35.6 & 5 \\
$3 p_{1 / 2}+3 p_{3 / 2}$ & 84.7 & 89.4 & 6 \\
$3 d_{3 / 2}+3 d_{5 / 2}$ & 29.5 & 31.6 & 7 \\
\hline
\end{tabular}

the $3 \ell j$ levels of the ion $\mathrm{Fe}^{16+}$. Comparison between our approximate and exact results for the Ne-like ion $\mathrm{Kr}^{26+}$ [31] suggests the uncertainty in $F_{2}$ to be $\simeq 4 \%$. Therefore, the corrected value for $\mathrm{Fe}^{16+}$ is $F_{2}=1.26$.

Besides, we verify by inspection relevant theoretical data used in [13] for normalizing the measured intensity of RR peaks by comparison with our RRCS calculations by the DF method. The DF values of RRCS $\sigma_{\mathrm{DF}}$ for the $3 s, 3 p$ and $3 d$ states at electron energy $E_{k}=964 \mathrm{eV}$ are listed in Table 2 together with RRCS calculated by the DS method $\sigma_{\mathrm{DS}}$ and differences $\Delta$ between the two calculations.

As is seen from Table 2, the DF method decreases RRCS, on average, by 6\%, as compared to the DS method. Thus, the correction associated with the inclusion of PRR channel (26\%) along with the use of the more appropriate DF method in the RRCS calculations (6\%) increases RRCS and, consequently, EIECS by $\sim 20 \%$. This resolved the contradiction between experimental and theoretical values of $\sigma_{\text {EIE. }}$.

\subsection{PRR Effect for Highly-Charged Tungsten Ions}

The agreement of our calculations with exact results [31] and, particularly, a good agreement between experimental and theoretical values of EIECS resulting from including the PRR effect in the RRCS calculations give good grounds for believing that the adopted approximation provide a reasonable estimation of the RRCS enhancement for highly-charged tungsten ions. We consider the PRR effect for tungsten ions with charges $24 \leq q \leq 64$. The $k$-dependence of $F_{n}$ is demonstrated in Figure 8 for representative tungsten ions. The enhancement factor $F_{4}$ is presented in the photon energy range $I_{4 s} \lesssim k \lesssim I_{3 d_{5 / 2}}$; the factor $F_{3}$ is given in the range $I_{3 s} \lesssim k \lesssim I_{2 p_{3 / 2}}$; and the factor $F_{2}$ for $k \gtrsim I_{2 s}$. Electron configurations of tungsten ions along with the states in which an electron is captured in the RR process are listed in Table 3.

As is evident from Figure 8, the enhancement factors $F_{3}$ and $F_{4}$ drop rapidly as the photon energy increases (see Equation (20)). All factors decrease gradually when the ion charge increases. For example, the maximum value of $F_{3}$ decreases from $17 \%$ for $\mathrm{W}^{24+}$ to $11 \%$ for $\mathrm{W}^{46+}$ in spite of the fact that the $3 s$, $3 p$ and $3 d$ subshells are closed in both ions. The largest enhancement factor is $F_{3}$ provided that the $3 d$ electrons are involved into polarization. The factor $F_{4}$ involving the closed $4 d$ subshells is not so large. For example, the maximum value of $F_{3}$ for $\mathrm{W}^{28+}$ equals $\sim 15 \%$, while $F_{4}$ equals $\sim 6 \%$, both the $3 d$ and $4 d$ shells being closed. Polarization of the $n s$ and $n p$ shells at $n=3,4$ results in a small effect, $F_{3}$ and $F_{4}$ being less than $3 \%$. For $\mathrm{W}^{56+}$ and $\mathrm{W}^{60+}$ where the $3 s$ and $3 p$ subshells are polarized, $F_{3} \lesssim 2.5 \%$ and $\lesssim 1.1 \%$, respectively. Calculations also showed that for $\mathrm{W}^{38+}$ and $\mathrm{W}^{42+}$ with polarization of the $4 s$ and $4 p$ shells, the maximum value of $F_{4}$ is $\sim 2 \%$ and $1.3 \%$, respectively. The factor $F_{2}$ associated 
with polarization of the $2 s$ and $2 p$ shells decreases gradually from $\sim 7 \%$ down to $\sim 3 \%$ when the photon energy increases. The factor also decreases with increasing the ion charge. It should be noted that, as is shown in Figure 6, our values of $F_{3}$ are overestimated at low energies as compared with the Hartree-Fock calculations in [31]. Because of this, it is quite possible that enhancement factors $F_{3}$ presented in Figure 8 for tungsten ions are also overestimated near the threshold.

Table 3. Electron configurations adopted for recombining tungsten ions given in Figure 8 and the electron state in which an electron is captured.

\begin{tabular}{ccc}
\hline Ion & Electron Configuration & Final State \\
\hline $\mathrm{W}^{24+}$ & {$[\mathrm{Kr}] 4 d_{3 / 2}^{4} 4 d_{5 / 2}^{6} 4 f_{5 / 2}^{4}$} & $4 f_{5 / 2}$ \\
$\mathrm{~W}^{28+}$ & {$[\mathrm{Kr}] 4 d_{3 / 2}^{4} 4 d_{5 / 2}^{6}$} & $5 s_{1 / 2}$ \\
$\mathrm{~W}^{34+}$ & {$[\mathrm{Kr}] 4 d_{3 / 2}^{4}$} & $4 d_{5 / 2}$ \\
$\mathrm{~W}^{46+}$ & {$[\mathrm{Ar}] 3 d_{3 / 2}^{4} 3 d_{5 / 2}^{6}$} & $4 s_{1 / 2}$ \\
$\mathrm{~W}^{56+}$ & {$[\mathrm{Ar}]$} & $3 d_{3 / 2}$ \\
$\mathrm{~W}^{64+}$ & {$[\mathrm{Ne}]$} & $3 s_{1 / 2}$ \\
\hline
\end{tabular}

The difference between $F_{3}$ and $F_{4}$ involving the $3 d$ and $4 d$ shells, respectively, is associated with the fact that the $3 d$ density in the interval $\left[0-r_{0}\right.$ ] (see Equations (20)-(22)) is considerably larger than the $4 d$ density. Relativistic electron densities for these shells of $\mathrm{W}^{28+}$ are compared in Figure 9 at the photon energies close to their ionization thresholds that determine different values of $r_{0}$. In spite of the fact that $r_{0}=0.183$ a.u. for the $3 d_{3 / 2}$ electron is less than $r_{0}=0.303$ a.u. for the $4 d_{3 / 2}$ electron in the case displayed in Figure 9, it is evident that the density, and hence, the integral value $N_{\text {out }}\left(r_{0}\right)$, is much larger for the $3 d_{3 / 2}$ shell. In line with this, as is seen from Figure 8, the maximum value of $F_{3}$ for $\mathrm{W}^{28+}$ considerably exceeds the maximum value of $F_{4}$.

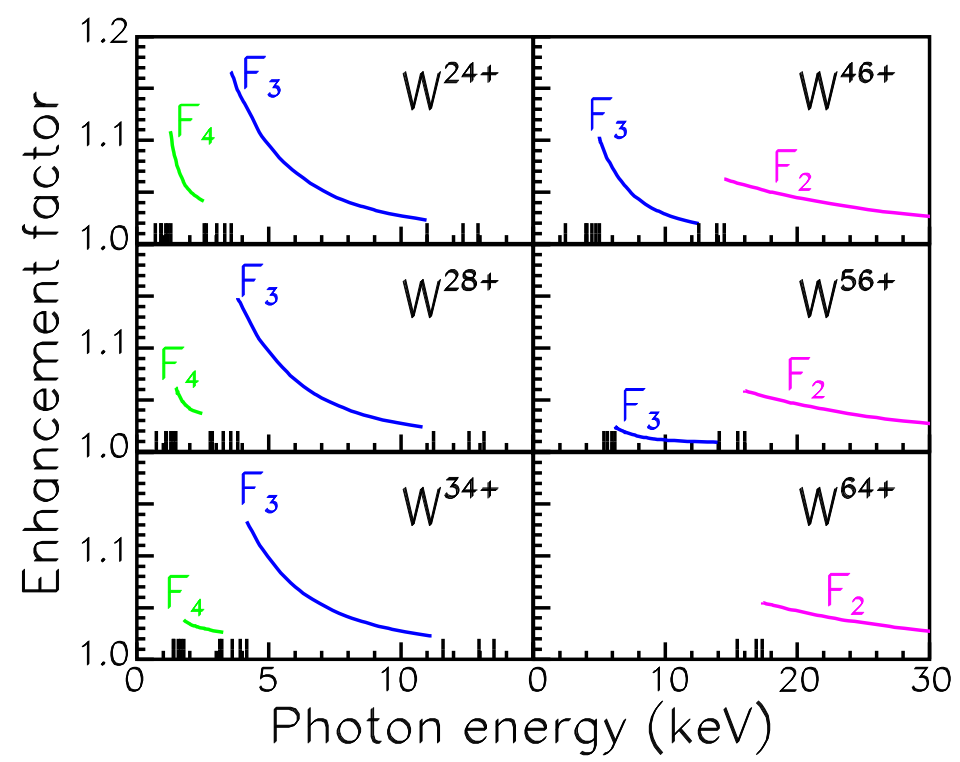

Figure 8. Enhancement factors $F_{n}$ for RR of representative tungsten ions with an electron captured in the lowest state. Green, $F_{4}$; blue, $F_{3}$; rose, $F_{2}$. Vertical lines denote ionization energies. 
As is clear from Table 3, enhancement factors in Figure 8 are presented for RR of tungsten ions with an electron captured in the lowest ion state. However, there is only a slight difference between $F_{n}$ for a capture in various electron states within the approximation used here. The enhancement factors for various final electron states in the photon energy ranges free from DR resonances are listed in Table 4. As is seen, the difference between $F_{n}$ at the electron capture in various states does not exceed $0.3 \%$.

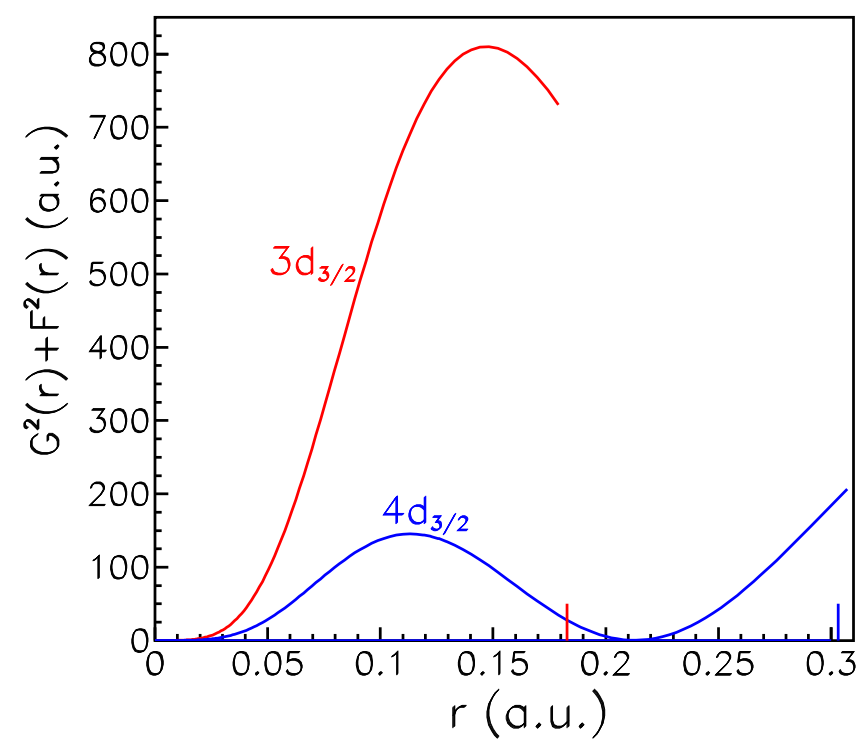

Figure 9. The electron density $G^{2}(r)+F^{2}(r)$ in the range $\left[0-r_{0}\right]$ for the $3 d_{3 / 2}$ (red) and $4 d_{3 / 2}$ (blue) shells of the ion $\mathrm{W}^{28+}$. Vertical lines denote values of $r_{0}$ for the $3 d_{3 / 2}$ (red) and $4 d_{3 / 2}$ (blue) shells.

Table 4. The percentage enhancement factor $\left(F_{n}-1\right) \times 100 \%$ for $\mathrm{RR}$ of $\mathrm{W}^{28+}$ with an electron captured in various states.

\begin{tabular}{cccccccc}
\hline $\mathbf{n}$ & $\boldsymbol{k}, \mathbf{k e V}$ & $\boldsymbol{5} \boldsymbol{s}$ & $\mathbf{4} \boldsymbol{f}_{\mathbf{5} / \mathbf{2}}$ & $\mathbf{5} \boldsymbol{d}_{\mathbf{3} \mathbf{2}}$ & $\mathbf{6} \boldsymbol{p}_{\mathbf{1 / 2}}$ & $\mathbf{5 g _ { \mathbf { 7 } / \mathbf { 2 } }}$ & $\mathbf{7 s}$ \\
\hline \multirow{3}{*}{3} & 3.82 & 14.8 & 15.0 & 14.7 & 14.8 & 14.8 & 14.7 \\
& 7.00 & 5.2 & 5.2 & 5.2 & 5.2 & 5.2 & 5.2 \\
& 11.23 & 2.2 & 2.2 & 2.2 & 2.2 & 2.2 & 2.2 \\
\hline \multirow{2}{*}{4} & 1.70 & 4.9 & 5.0 & 4.9 & 4.9 & 4.9 & 4.8 \\
& 2.79 & 3.5 & 3.5 & 3.4 & 3.4 & 3.4 & 3.3 \\
\hline
\end{tabular}

Consequently, we showed that the simple analytical "stripping" approximation provides a reasonable estimation of the PRR enhancement factor for RRCS. The approximation was used for an assessment of the PRR enhancement for RR with a capture of an electron in the $3 \ell j$ levels of Fe XVII. Enhancement factors for highly-charged tungsten ions were calculated. It was obtained that the most enhancement occurs when the $3 d_{3 / 2}$ and $3 d_{5 / 2}$ electrons are involved in the polarization. The enhancement factor decreases with increasing of the photon energy. The factor depends on the principal quantum number of polarized shells and on the ion charge. This factor should be taken into account in the RRCS calculations. 


\section{Ions in Dense Laser and Fusion Plasmas}

\subsection{Average-Atom Model}

To study the electron structure of ions in LTE plasmas, the code PLASMASATOM has been designed on the basis of our computer program complex RAINE [16-18]. The code PLASMASATOM is based on the average-atom model. The model has been applied in the Los Alamos code INFERNO [19,20], code PURGATORIO [21] and the more advanced code PARADISIO [22].

In the average-atom model, the plasma is taken to consist of the neutral Wigner-Seitz (WS) cells [34,35]. Each of them contains a nucleus with a charge $Z$ and $Z$ bound and continuum electrons. The bound wave function and its derivative coincide with those of a neighboring atom. The continuum density is finite at the WS cell boundary and merges into the uniform free-electron density outside the cell. Therefore, we treat an isolated neutral cell in a local thermodynamic average sense, neglecting the interaction of the cell with other ones. The radius of the WS cell $R_{\mathrm{WS}}$ is determined from the material density and atomic weight.

In the following studies, we used the relativistic DS method where an electron is assumed to satisfy the system of the Dirac central-field equations:

$$
\begin{aligned}
\frac{d G(r)}{d r} & =-\frac{\kappa}{r} G(r)+[E+1-V(r)] F(r) \\
\frac{d F(r)}{d r} & =\frac{\kappa}{r} F(r)-[E-1-V(r)] G(r)
\end{aligned}
$$

Here, $E$ is the total electron energy, and $V(r)$ is the electron potential energy. The potential with the exchange term in the local density approximation may be written as:

$$
V(r)= \begin{cases}-\frac{\alpha Z}{r}+\frac{\alpha}{r}\left[\int_{0}^{r} 4 \pi r^{2} \rho(r) d r+r \int_{r}^{R_{\mathrm{WS}}} 4 \pi r \rho(r) d r\right]-\alpha\left[\frac{3}{\pi} \rho(r)\right]^{1 / 3} \text { at } \quad r \leq R_{\mathrm{WS}} \\ 0 & \text { at } \quad r>R_{\mathrm{WS}}\end{cases}
$$

where $\rho(r)$ is the total electron density:

$$
\rho(r)=\rho_{b}(r)+\rho_{c}(r)
$$

The bound density contribution $\rho_{b}(r)$ is written as:

$$
4 \pi r^{2} \rho_{b}(r)=\sum_{i}\left(2 j_{i}+1\right) f_{i}\left(\varepsilon_{i}, \mu\right)\left[G_{i}^{2}(r)+F_{i}^{2}(r)\right]
$$

where the summation is over all bound states, and the Fermi-Dirac factor $f_{i}\left(\varepsilon_{i}, \mu\right)$ is given by the Fermi distribution:

$$
f_{i}\left(\varepsilon_{i}, \mu\right)=\left[1+\exp \left(\frac{\varepsilon_{i}-\mu}{k_{\beta} T}\right)\right]^{-1}
$$

Here, $\varepsilon_{i}=1-E<0$ is the electron binding energy, $\mu$ is the chemical potential and $k_{\beta} T$ is the temperature. The occupation number of the $\mathrm{i}$-th level is determined by:

$$
N_{i}=\left(2 j_{i}+1\right) f_{i}\left(\varepsilon_{i}, \mu\right)
$$


Bound electron wave functions are normalized so that:

$$
\int_{0}^{R_{\mathrm{WS}}}\left[G_{i}^{2}(r)+F_{i}^{2}(r)\right] d r=1
$$

The continuum density contribution $\rho_{c}(r)$ has the form:

$$
4 \pi r^{2} \rho_{c}(r)=\int_{0}^{\infty} d \varepsilon f(\varepsilon, \mu) \sum_{\kappa= \pm 1}^{ \pm \infty} 2|\kappa|\left[G_{\kappa}^{2}(r)+F_{\kappa}^{2}(r)\right]
$$

Here, $\varepsilon=E-1>0$, and the associated Fermi-Dirac factor $f(\varepsilon, \mu)$ is given by:

$$
f(\varepsilon, \mu)=\left[1+\exp \left(\frac{\varepsilon-\mu}{k_{\beta} T}\right)\right]^{-1}
$$

Continuum wave functions are normalized per unit energy interval, so that:

$$
\lim _{r \rightarrow \infty}\left[G_{\kappa}^{2}(r)+\frac{E+1}{E-1} F_{\kappa}^{2}(r)\right]=\frac{1}{\pi} \sqrt{\frac{E+1}{E-1}}
$$

The chemical potential $\mu$ appearing in Equations (28) and (32) is determined provided that the cell with the radius $R_{\mathrm{WS}}$ is electrically neutral:

$$
F(\mu)=Z-4 \pi \int_{0}^{R_{\mathrm{WS}}} r^{2} \rho(r) d r=0
$$

The sum over $\kappa$ in Equation (31) converges slowly. Because of this, to eliminate the need of a direct calculations of the sum, we transform Equation (31) by the method, which makes it possible to perform the summation over $\kappa$ not to infinity, but only for a few values of $|\kappa| \leq\left|\kappa_{\max }\right|$ [36]. In the case of the DS method and for our notations, Equation (31) may be rearranged by the following way. Where the influence of the potential $V(r)$ is negligible, the continuum wave function normalized according to Equation (33) may be written as:

$$
\begin{aligned}
G_{\kappa}(r) & =N_{\varepsilon} p r\left[\cos \delta j_{\ell}(p r)+\sin \delta y_{\ell}(p r)\right] \\
F_{\kappa}(r) & =\frac{\kappa}{|\kappa|} N_{\varepsilon} p r \sqrt{\frac{E-1}{E+1}}\left[\cos \delta j_{\bar{\ell}}(p r)+\sin \delta y_{\bar{\ell}}(p r)\right]
\end{aligned}
$$

where $\delta(r)$ is the phase shift, $p=\sqrt{E^{2}-1}, N_{\varepsilon}=\sqrt{\frac{E+1}{\pi p}}$ is the normalization factor and $j_{\ell}$ and $y_{\ell}$ are the spherical Bessel functions of the first and second kind, respectively.

Setting in Equations (35) $\delta=0$, we arrive to functions $G(r)$ and $F(r)$ for a free wave:

$$
\begin{aligned}
G_{\delta=0, \kappa}(r) & =N_{\varepsilon} p r j_{\ell}(p r) \\
F_{\delta=0, \kappa}(r) & =\frac{\kappa}{|\kappa|} \sqrt{\frac{E-1}{E+1}} N_{\varepsilon} p r j_{\bar{\ell}}(p r)
\end{aligned}
$$


Further, we replace the summation over $\kappa$ in Equation (31) by two sums:

$$
\sum_{\kappa= \pm 1}^{ \pm \infty} 2|\kappa|\left[G_{\kappa}^{2}(r)+F_{\kappa}^{2}(r)\right]=\sum_{\kappa= \pm 1}^{ \pm\left|\kappa_{\max }\right|} 2|\kappa|\left[G_{\kappa}^{2}(r)+F_{\kappa}^{2}(r)\right]+\sum_{\kappa= \pm\left(\left|\kappa_{\max }\right|+1\right)}^{ \pm \infty} 2|\kappa|\left[G_{\kappa}^{2}(r)+F_{\kappa}^{2}(r)\right]
$$

In addition, to accelerate the sum convergence, we subtract from the real density $\left(G_{\kappa}^{2}+F_{\kappa}^{2}\right)$ the relevant density for a free wave $\left(G_{\delta=0, \kappa}^{2}+F_{\delta=0, \kappa}^{2}\right)$ in each $\kappa$-term of the first sum in the right part of Equation (37). To compensate these terms and to include the second sum in Equation (37), we add the total sum of such terms for $\pm 1 \leq \kappa \leq \pm \infty$. Then, the expression for $\rho_{c}(r)$ takes the form:

$$
\begin{aligned}
\rho_{c}(r) & =\int_{0}^{\infty} d \varepsilon f(\varepsilon, \mu)\left\{\sum_{\kappa= \pm 1}^{ \pm\left|\kappa_{\max }\right|} \frac{|\kappa|}{2 \pi}\left[\frac{G_{\kappa}^{2}(r)+F_{\kappa}^{2}(r)}{r^{2}}-N_{\varepsilon}^{2} p^{2}\left(j_{\ell}^{2}(p r)+\frac{E-1}{E+1} j_{\bar{\ell}}^{2}(p r)\right)\right]\right. \\
& \left.+\frac{N_{\varepsilon}^{2} p^{2}}{2 \pi}\left[\sum_{\kappa= \pm 1}^{ \pm \infty}|\kappa| j_{\ell}^{2}(p r)+\frac{E-1}{E+1} \sum_{\kappa= \pm 1}^{ \pm \infty}|\kappa| j_{\bar{\ell}}^{2}(p r)\right]\right\}
\end{aligned}
$$

Terms in the second line of Equation (38) compensate the sum over $\pm 1 \leq \kappa \leq \pm\left|\kappa_{\max }\right|$ for a free wave, which we have subtracted, as well as include approximately the sum over $\pm\left(\left|\kappa_{\max }\right|+1\right) \leq \kappa \leq$ $\pm \infty$. It is easy to check that:

$$
\begin{aligned}
& \sum_{\kappa= \pm 1}^{ \pm \infty}|\kappa| j_{\ell}^{2}(p r)=\sum_{\ell=0}^{\infty}(2 \ell+1) j_{\ell}^{2}(p r)=1 \\
& \sum_{\kappa= \pm 1}^{ \pm \infty}|\kappa| j_{\bar{\ell}}^{2}(p r)=\sum_{\ell=0}^{\infty}(2 \ell+1) j_{\ell}^{2}(p r)=1
\end{aligned}
$$

Taking into consideration Equations (33), (39) and (40), we arrive at the following expression for the continuum density:

$$
\rho_{c}(r)=\int_{0}^{\infty} d \varepsilon f(\varepsilon, \mu)\left\{\sum_{\kappa= \pm 1}^{ \pm\left|\kappa_{\max }\right|} \frac{|\kappa|}{2 \pi}\left[\frac{G_{\kappa}^{2}(r)+F_{\kappa}^{2}(r)}{r^{2}}-\frac{(E+1) p}{\pi}\left(j_{\ell}^{2}(p r)+\frac{E-1}{E+1} j_{\bar{\ell}}^{2}(p r)\right)\right]+\frac{E p}{\pi^{2}}\right\}
$$

It is instructive to evaluate how many terms have to be taken into account in sum over $\kappa$ in Equation (41). We present in Table 5 results obtained with various values of $\left|\kappa_{\max }\right|$ for the iron ion at temperature $100 \mathrm{eV}$ and $R_{\mathrm{WS}}=2.672$ a.u. Data of Table 5 demonstrate differences in a third or fourth significant digit of the $\varepsilon_{i}, N_{i}$ and $\mu$ magnitudes obtained in calculations having regard to $\left|\kappa_{\max }\right|=10$ and $\left|\kappa_{\max }\right|=15$. This means that the difference between the two calculations is less than $0.5 \%$. We checked that further increasing $\left|\kappa_{\max }\right|$ has no influence on the results. Therefore, the value $\left|\kappa_{\max }\right|=10$ is slightly lacking to give a high accuracy, while $\left|\kappa_{\max }\right|=15$ is quite enough. Consequently, adoption of Equation (41) for $\rho_{c}(r)$ permits one to restrict $\left|\kappa_{\max }\right|=15$, while the direct summation in Equation (31) requires several tens of $\kappa$-terms to reach the same accuracy.

The integral over $\varepsilon$ in Equation (41) is evaluated up to $\varepsilon_{\max }$, where $\varepsilon_{\max }$ is chosen so that the Fermi-Dirac factor is small:

$$
f\left(\varepsilon_{\max }, \mu\right) \leq \delta_{\varepsilon}
$$


where $\delta_{\varepsilon}=10^{-8}$. The integrand is calculated for $10^{3}$ equidistant points $\varepsilon$ in the interval $\left[0-\varepsilon_{\max }\right]$. This energy grid is used for calculation of the integral in Equation (41) by the Simpson method. Calculations where the continuum density is based on Equations (31)-(42) will be refereed to as the DS-DS model.

Table 5. Binding energies $\varepsilon_{i}$, occupation numbers $N_{i}, N_{\text {bound }}$ (see Equation (53) below), the charge $q$ and the chemical potential $\mu$ calculated for various $\left|\kappa_{\max }\right|$ for the iron ion at $k_{\beta} T=100 \mathrm{eV}$ and $R_{\mathrm{WS}}=2.672$ a.u.

\begin{tabular}{cccccc}
\hline \multirow{2}{*}{ Shell } & \multicolumn{2}{c}{$\left|\boldsymbol{\kappa}_{\max }\right|=\mathbf{1 0}$} & & \multicolumn{2}{c}{$\left|\boldsymbol{\kappa}_{\max }\right|=\mathbf{1 5}$} \\
\cline { 2 - 3 } \cline { 5 - 6 } & $\boldsymbol{\varepsilon}_{\boldsymbol{i}}(\mathbf{e V})$ & $\boldsymbol{N}_{\boldsymbol{i}}$ & & $\boldsymbol{\varepsilon}_{\boldsymbol{i}}(\mathbf{e V})$ & $\boldsymbol{N}_{\boldsymbol{i}}$ \\
\hline $1 s$ & $-7,110.09$ & 2.0000 & & $-7,110.47$ & 2.0000 \\
$2 s$ & -931.53 & 1.9986 & & -931.91 & 1.9986 \\
$2 p_{1 / 2}$ & -822.19 & 1.9957 & & -822.84 & 1.9957 \\
$2 p_{3 / 2}$ & -809.54 & 3.9903 & & -809.92 & 3.9903 \\
$3 s$ & -169.74 & 0.8141 & & -170.02 & 0.8122 \\
$3 p_{1 / 2}$ & -136.00 & 0.6576 & & -136.27 & 0.6559 \\
$3 p_{3 / 2}$ & -133.85 & 1.2963 & & -134.12 & 1.2928 \\
$3 d_{3 / 2}$ & -80.11 & 0.8752 & & -80.38 & 0.8725 \\
$3 d_{5 / 2}$ & -79.76 & 1.3094 & & -80.06 & 1.3054 \\
$4 s$ & -16.98 & 0.2594 & & -17.06 & 0.2580 \\
\hline$N_{\text {bound }}$ & & 15.1966 & & 15.1814 \\
$q$ & & 10.8015 & & 10.8186 \\
$\mu(\mathrm{eV})$ & -207.37 & & -208.04 & \\
\hline
\end{tabular}

To simplify and significantly accelerate the computational procedure, we also elaborated another version of code PLASMASATOM, where $\rho_{c}$ is evaluated within the framework of the semi-classical Thomas-Fermi (TF) approximation according to [34]. In this case, a continuum density is written in atomic units as follows:

$$
\rho_{c}(r)=\frac{\left(2 m k_{\beta} T\right)^{3 / 2}}{2 \pi^{2}} I_{1 / 2}(b, x)
$$

Here, $I_{1 / 2}(b, x)$ is the incomplete Fermi integral:

$$
I_{1 / 2}(b, x)=\int_{b}^{\infty} \frac{y^{1 / 2} d y}{1+\exp (y-x)}
$$

where:

$$
\begin{gathered}
x=\left\{\mu-\left[V(r)-V_{\mathrm{ex}}(r)\right]\right\} / k_{\beta} T \\
b=-\left[V(r)-V_{\mathrm{ex}}(r)\right] / k_{\beta} T
\end{gathered}
$$

and $V_{\mathrm{ex}}(r)$ is the exchange term of the potential $V(r)$. Calculations where the continuum density is based on Equations (43)-(46) will be refereed to as the DS-TF model.

In the both models, the SCF values of $V(r), \rho(r)$ and $\mu$ are found by the iterative method. The process starts from calculations for a neutral atom by the DS method without regard for a temperature. The initial 
potential $V^{0}(r)$ constructed from the DS bound wave functions allows us to determine the initial density. Two initial values of the chemical potential $\mu_{0}$ and $\mu_{0}^{\prime}$ have to be specified so that:

$$
F\left(\mu_{0}\right) F\left(\mu_{0}^{\prime}\right)<0
$$

Further, on the $n$-th iteration, we calculate a root of Equation (34) $\mu_{n}$. Knowledge of a new value of $\mu_{n}$ permits finding Fermi-Dirac factors $f_{i}\left(\varepsilon_{i}, \mu\right)$ and $f(\varepsilon, \mu)$, then new densities $\rho_{b}(r), \rho_{c}(r)$ and $\rho(r)$, which permit, in turn, to determine a new potential $V^{n+1}(r)$. The iterative process is accomplished when the following condition is fulfilled:

$$
\max _{r}\left|V^{n+1}(r)-V^{n}(r)\right|<\delta_{V}
$$

The accuracy of SCF calculations is chosen as $\delta_{V}=10^{-5}$.

In a general case, the iterative process is unstable. Because of this, the initial potential for the $(n+1)$-th iteration $V^{(n+1) i}(r)$ is determined using the initial and final potentials for previous $n$-th and $(n-1)$-th iterations in the following manner. If the iteration number $(n+1)$ is odd, the initial potential is determined as:

$$
V^{(n+1) i}(r)=A V^{n i}(r)+(1-A) V^{n f}(r)
$$

where the mixing coefficient $A$ is prescribed within the limits $0.2 \leq A \leq 0.9$.

If the iteration number $(n+1)$ is even, the initial potential is calculated using the following scheme (see [16] and the references therein):

$$
V^{(n+1) i}(r)=\left\{\begin{array}{lr}
V^{n f}(r) & \text { for } B(r)<0 \\
\bar{V}(r) & \text { for } 0 \leq B(r) \leq A \\
A V^{n i}(r)+(1-A) V^{n f}(r) \text { for } B(r)>A
\end{array}\right.
$$

Here:

$$
B(r)=\frac{V^{n f}(r)-\bar{V}(r)}{V^{n f}(r)-V^{n i}(r)}
$$

and:

$$
\bar{V}(r)=\frac{V^{(n-1) i}(r) V^{n f}(r)-V^{n i}(r) V^{(n-1) f}(r)}{\left[V^{n f}(r)-V^{(n-1) f}(r)\right]-\left[V^{n i}(r)-V^{(n-1) i}(r)\right]}
$$

If the iterative process diverges just the same, the mixing coefficient $A$ should be increased.

\subsection{Comparison with Previous Calculations}

Our results were verified by comparing with calculations [34] for iron. The bound, continuum and total densities for the iron ion in plasmas with the normal density at $k_{\beta} T=100 \mathrm{eV}$ are shown in Figure 10 .

As is seen from Figure 10, our results (red curves) are very close to the results from [34] (blue curves). The slight differences occur in $\rho_{b}$ in ranges of maxima and minima where electron wave functions are usually very sensitive to all details of calculations and in $\rho_{c}$ near the very WS boundary. 
Next, we compare our final results for the iron ion with calculations [34]. Presented in Table 6 are the spectrum of binding energies $\varepsilon_{i}$, level populations $N_{i}$ (Equation (29)), the chemical potential $\mu$, the ion charge $q$ and a number of bound electrons $N_{\text {bound }}$ in the resulting ion:

$$
N_{\text {bound }}=\sum_{i} N_{i}=Z-q
$$

Our data were obtained using the DS-TF and DS-DS models. One can see that the results calculated by the DS-TF model correlate with data from [34], where the same model has been adopted. Our binding energies are in excellent agreement with those from [34]. The largest difference for the binding energy of the valence $4 s$ level is likely to be due to different boundary conditions. However, the difference in level occupation numbers reaches $\sim 12 \%$. In addition, data of Table 6 allow one to compare results obtained within the DS-DS and DS-TF models. As is seen, there is a minor difference between the two calculations. The largest difference $(\sim 5 \%)$ occurs for the binding energy of the valence $4 s$ shell. Values of $\mu$ obtained in the two models differ by $\lesssim 0.3 \%$, and values of the charge $q$ only by $\sim 0.2 \%$. It should be noted that all levels and the chemical potential become lower, as well as the charge increases when passing from the DS-TF model to the DS-DS one.

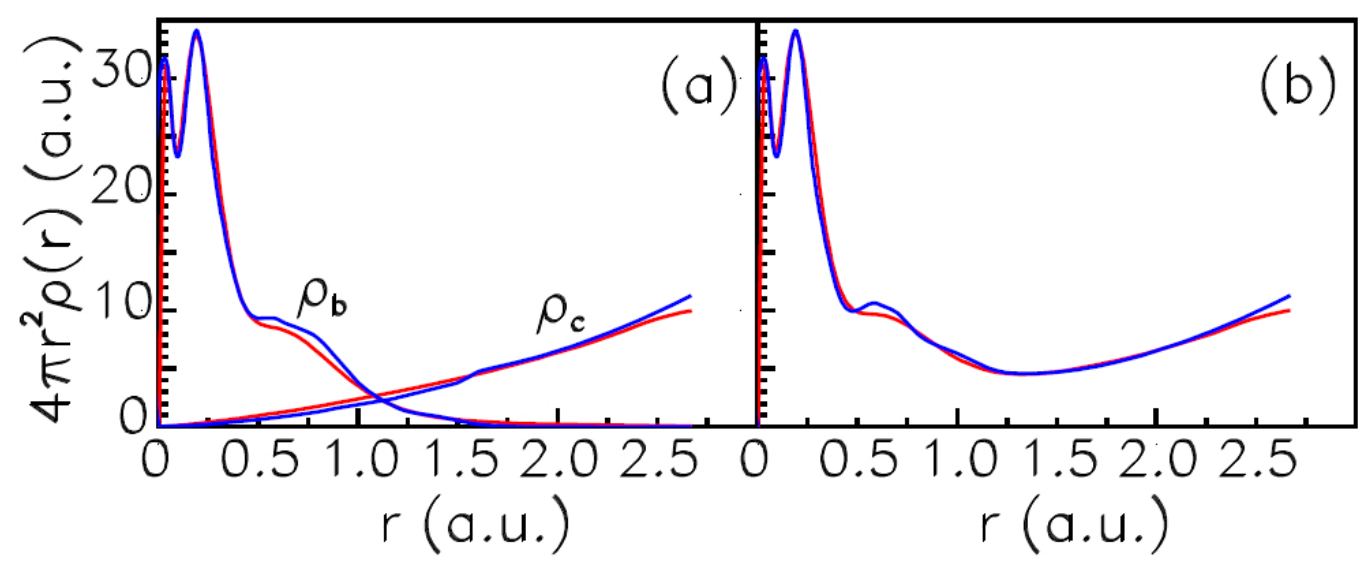

Figure 10. The bound and continuum electron densities (a) and the total density (b) calculated using the DS-Thomas-Fermi (TF) model for the iron ion in laser plasmas at temperature $k_{\beta} T=100 \mathrm{eV}$ and the normal density $7.87 \mathrm{~g} / \mathrm{cm}^{3}, R_{\mathrm{Ws}}=2.67$ a.u. Red, present calculations; blue, calculations [34].

As is well known, the DS and TF continuum densities diverge drastically, the DS density $\rho_{c}(r)$ being an oscillating function, while the $\mathrm{TF} \rho_{c}(r)$ is a quite smooth function. Nevertheless, the results obtained using the DS-DS and DS-TF models are very close to each other.

In addition, we compare our ion charge $q$ for iron in three cases listed in Table 7 with mean ionization stages $\langle q\rangle$ obtained by eight groups from Los Alamos, Livermore and with the data from Opacity Project (OPAC collaboration). The results have been calculated with different eleven codes to prepare LULI (Laboratoire pour L'Utilization des Lasers Intenses) 2010 experiments [37]. The difference in the mean ionization stage obviously implies the discrepancy between the frequency-dependent opacity. Therefore, the data are of importance for astrophysics. 
As is shown in Figure 11, our values of $q$ (red asterisks versus codes with numbers 12 and 3) correlate well with previous calculations. Besides, the data of Table 7 show that our results are in excellent agreement with the best results [37] of $\langle q>$ from OP. The largest difference is $4 \%$.

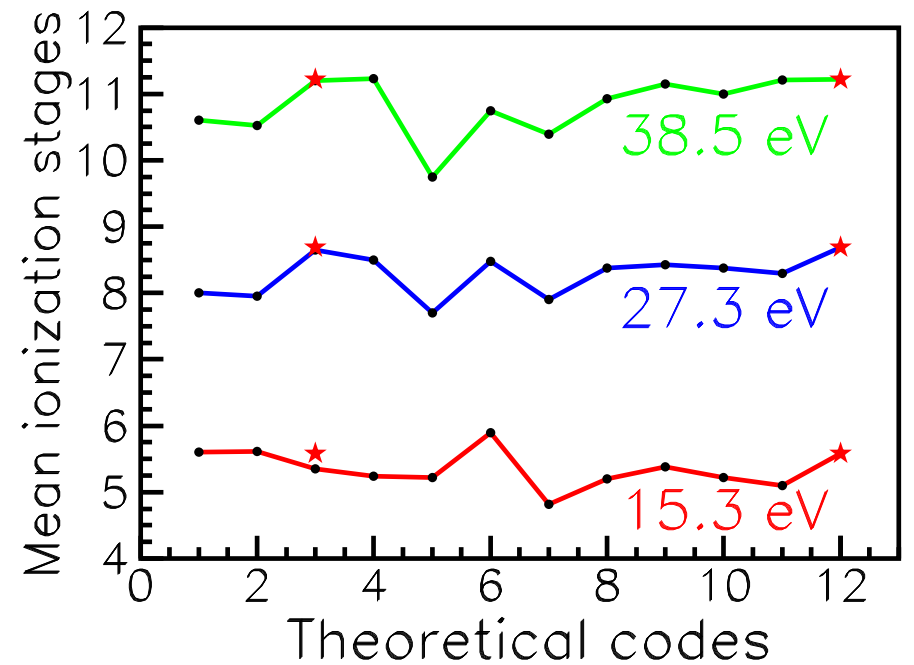

Figure 11. Iron mean ionization stages $\langle q\rangle$ obtained by various codes. Codes used are: 1: FLYCHK (NLTE ); 2: FLYCHK (LTE); 3: OP $(\cdot)$ and present results $(\star)$; 4: STA ; 5: AA $-Z_{P}$; 6: AA- $Z_{M}$; 7: CASSANDRA ; 8: OPAS ; 9: SCO(rel.) ; 10: SCO-RCG ; 11: LEDCOP ; 12: present calculations $(\star)$, PLASMASATOM. Codes 5, 6 and 12 are LTE average atom ionization models. Figure is taken from [37] with our results added for comparison.

Table 6. Spectrum of binding energies $\varepsilon_{i}$, level populations $N_{i}, N_{\text {bound }}$, the charge $q$ and the chemical potential $\mu$ for the iron ion at $k_{\beta} T=100 \mathrm{eV}$ and $R_{\mathrm{WS}}=2.67$ a.u.

\begin{tabular}{|c|c|c|c|c|c|c|}
\hline \multirow{3}{*}{ Shell } & \multicolumn{4}{|c|}{ Present Calculations } & \multirow{2}{*}{\multicolumn{2}{|c|}{$\frac{\text { Calculations [34] }}{\text { DS-TF }}$}} \\
\hline & \multicolumn{2}{|c|}{ DS-DS } & \multicolumn{2}{|c|}{ DS-TF } & & \\
\hline & $\varepsilon_{i}(\mathrm{eV})$ & $N_{i}$ & $\varepsilon_{i}(\mathrm{eV})$ & $N_{i}$ & $\varepsilon_{i}(\mathrm{eV})$ & $N_{i}$ \\
\hline $1 s$ & $-7,110.47$ & 2.0000 & $-7,110.04$ & 2.0000 & $-7,109.00$ & 2.0000 \\
\hline $2 s$ & -931.91 & 1.9986 & -931.50 & 1.9986 & -930.76 & 1.9988 \\
\hline $2 p_{1 / 2}$ & -822.84 & 1.9957 & -822.16 & 1.9957 & -821.40 & 1.9964 \\
\hline $2 p_{3 / 2}$ & -809.92 & 3.9903 & -809.51 & 3.9903 & -808.75 & 3.9919 \\
\hline $3 s$ & -170.02 & 0.8122 & -169.77 & 0.8147 & -169.96 & 0.9087 \\
\hline $3 p_{1 / 2}$ & -136.27 & 0.6559 & -136.03 & 0.6581 & -136.19 & 0.7453 \\
\hline $3 p_{3 / 2}$ & -134.12 & 1.2928 & -133.88 & 1.2973 & -134.04 & 1.4707 \\
\hline $3 d_{3 / 2}$ & -80.38 & 0.8725 & -80.08 & 0.8757 & -80.14 & 1.0131 \\
\hline $3 d_{5 / 2}$ & -80.06 & 1.3054 & -79.76 & 1.3103 & -79.81 & 1.5159 \\
\hline $4 s$ & -17.06 & 0.2580 & -16.14 & 0.2577 & -9.55 & 0.2868 \\
\hline$N_{\text {bound }}$ & & 15.1814 & & 15.1985 & & 15.9277 \\
\hline$q$ & & 10.8186 & & 10.8015 & & 10.0723 \\
\hline$\mu(\mathrm{eV})$ & -208.04 & & -207.27 & & -188.27 & \\
\hline
\end{tabular}


Table 7. Comparison of ionization stages $q$ for Fe obtained in present calculations with mean ionization stages $<q>$ from OP [38].

\begin{tabular}{ccccc}
\hline Case & $\boldsymbol{k}_{\boldsymbol{\beta}} \boldsymbol{T}(\mathrm{eV})$ & $\boldsymbol{\rho}\left(\mathbf{m g} / \mathbf{c m}^{\mathbf{3}}\right)$ & $\boldsymbol{q}$, Present & $\langle\boldsymbol{q}>$, OP \\
\hline 1 & 15.3 & 5.48 & 5.58 & 5.35 \\
2 & 27.3 & 3.39 & 8.69 & 8.65 \\
3 & 38.5 & 2.63 & 11.22 & 11.2 \\
\hline
\end{tabular}

We also compared results for the heavy uranium ion with those obtained in [22] using code PARADISIO. In Figure 12, the number of bound electrons $N_{\text {bound }}$ (Equation (53)) versus a temperature is presented for the uranium ion in plasmas with the density $\rho=0.01 \mathrm{~g} / \mathrm{cm}^{3}\left(N_{\text {ion }}=2.5 \cdot 10^{19} \mathrm{~cm}^{-3}\right)$. As is seen, our calculation (red curve) is in excellent agreement with the previous results (blue dashed curve) in the wide temperature range $0.1 \mathrm{eV} \leq k_{\beta} T \leq 10 \mathrm{keV}$.

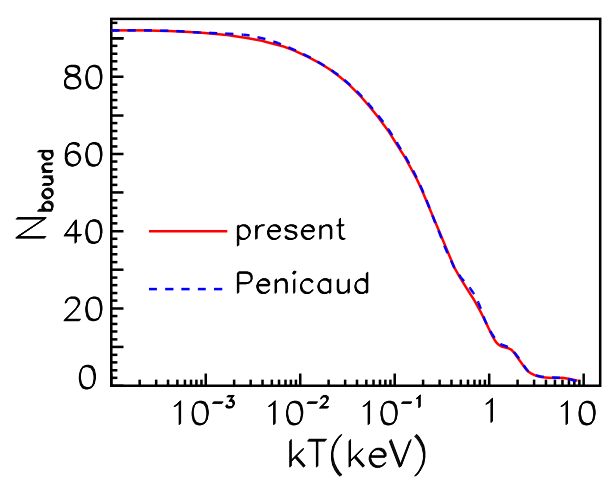

Figure 12. A number of bound electrons $N_{\text {bound }}\left(k_{\beta} T\right)$ for the uranium ion. Red solid, present calculations; blue dashed, results obtained in [22]. The plasma density is $\rho=0.01 \mathrm{~g} / \mathrm{cm}^{3}$.

\subsection{Results for Tungsten Ions}

New calculations were performed within the DS-DS model for the tungsten ion in laser plasmas of two densities $\rho_{1}=1.93 \mathrm{~g} / \mathrm{cm}^{3}$ (the ion density $N_{\text {ion }}=6.3 \times 10^{21} \mathrm{~cm}^{-3}$ ) and $\rho_{2}=0.01 \mathrm{~g} / \mathrm{cm}^{3}$ $\left(N_{\text {ion }}=3.3 \times 10^{19} \mathrm{~cm}^{-3}\right)$. The spectrum of energies $\varepsilon_{i}$ and level occupation numbers $N_{i}$ are given in Table 8 for the tungsten ion in plasmas with densities $\rho_{1}$ and the associated value of $R_{\mathrm{WS}}=6.339$ a.u. at temperatures $100 \mathrm{eV}$ and $1,000 \mathrm{eV}$, as well as for $\rho_{2}$ and $R_{\mathrm{WS}}=36.635$ a.u. at temperature $100 \mathrm{eV}$. Comparing data for $\rho_{1}$ at different temperatures, it may be noted that the ion compresses when the plasmas temperature increases, i.e., the levels become deeper and the outer shell occupation numbers decrease. As is seen from comparison data for various densities at $k_{\beta} T=100 \mathrm{eV}$, the ion compresses when the plasmas density decreases. As is seen from Table 8, high electron states contribute significantly at the higher density and lower temperature. For example, the $5 f, 5 g, 6 s, 6 p, 6 d, 6 f$ and $7 d$ levels have occupation numbers from $\sim 0.1-\sim 0.5$ in the case of $\rho_{1}$ and $k_{\beta} T=100 \mathrm{eV}$. Occupation numbers for all levels decrease with the temperature increasing and the density decreasing. 
The temperature dependence of $N_{\text {bound }}$ is presented in Figure 13 for the two densities. The blue curve refers to $\rho_{1}$ and the red curve to $\rho_{2}$. A comparison between the two curves gives an idea of the plasmas density dependence. As is seen, the red and blue curves are not too different, even though the associated densities differ by $\sim 200$-times. Increasing of a plasmas density shifts the curve $N_{\text {bound }}\left(k_{\beta} T\right)$ to higher temperatures.

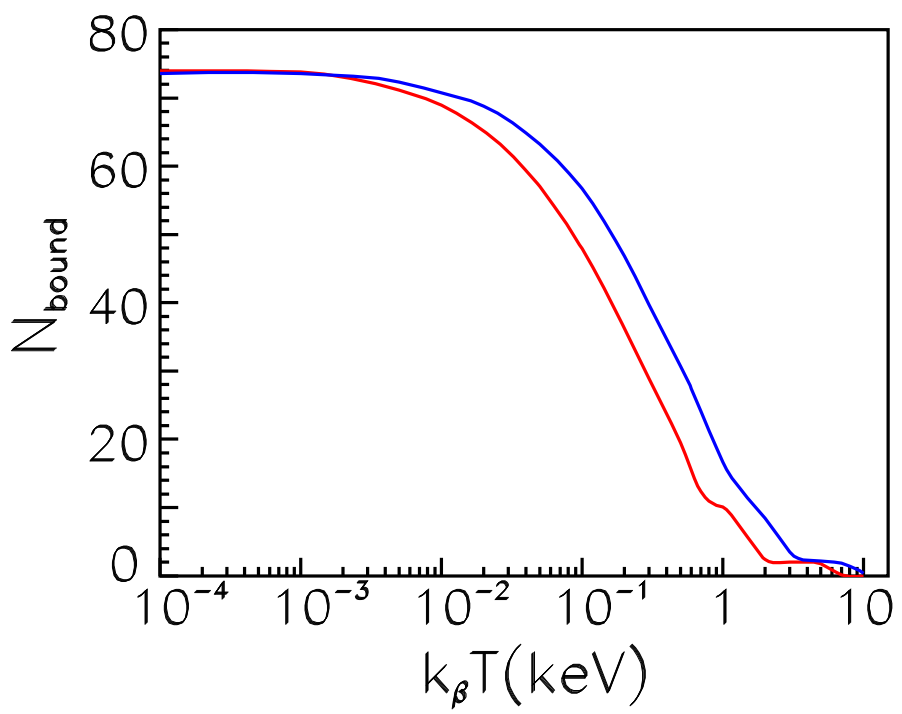

Figure 13. A number of bound electrons $N_{\text {bound }}\left(k_{\beta} T\right)$ for the tungsten ion in plasmas of various densities. Blue, $\rho_{1}=1.93 \mathrm{~g} / \mathrm{cm}^{3}$; red, $\rho_{2}=0.01 \mathrm{~g} / \mathrm{cm}^{3}$.

Table 8. Spectrum of energies $\varepsilon_{i}$ and level populations $N_{i}$ for the tungsten ion in plasmas with densities $\rho_{1}=1.93 \mathrm{~g} / \mathrm{cm}^{3}, R_{\mathrm{WS}}=6.339$ a.u. and $\rho_{2}=0.01 \mathrm{~g} / \mathrm{cm}^{3}, R_{\mathrm{WS}}=36.635$ a.u.

\begin{tabular}{|c|c|c|c|c|c|c|}
\hline \multirow{3}{*}{$\frac{\rho\left(\mathrm{g} / \mathrm{cm}^{3}\right)}{k_{\beta} T(\mathrm{eV})} \frac{\text { Shell }}{}$} & \multicolumn{4}{|c|}{1.93} & \multirow{2}{*}{\multicolumn{2}{|c|}{$\begin{array}{c}0.01 \\
100\end{array}$}} \\
\hline & \multicolumn{2}{|c|}{100} & \multicolumn{2}{|c|}{1000} & & \\
\hline & $\varepsilon_{i}(\mathbf{e V})$ & $N_{i}$ & $\varepsilon_{i}(\mathrm{eV})$ & $N_{i}$ & $\varepsilon_{i}(\mathbf{e V})$ & $N_{i}$ \\
\hline $1 s$ & $-69,722.54$ & 2.0000 & $-73,417.13$ & 2.0000 & $-70,164.51$ & 2.0000 \\
\hline $2 s$ & $-12,372.45$ & 2.0000 & $-15,833.79$ & 1.9999 & $-12,814.53$ & 2.0000 \\
\hline $2 p_{1 / 2}$ & $-11,855.50$ & 2.0000 & $-15,375.08$ & 1.9998 & $-12,297.85$ & 2.0000 \\
\hline $2 p_{3 / 2}$ & $-10,501.65$ & 4.0000 & $-13,987.02$ & 3.9982 & $-10,943.37$ & 4.0000 \\
\hline $3 s$ & $-3,166.07$ & 2.0000 & $-6,021.08$ & 0.8729 & $-3,599.70$ & 2.0000 \\
\hline $3 p_{1 / 2}$ & $-2,935.18$ & 2.0000 & $-5,847.31$ & 0.7885 & $-3,369.64$ & 2.0000 \\
\hline $3 p_{3 / 2}$ & $-2,641.56$ & 4.0000 & $-5,486.22$ & 1.2483 & $-3,074.47$ & 4.0000 \\
\hline $3 d_{3 / 2}$ & $-2,250.91$ & 4.0000 & $-5,240.91$ & 1.0478 & $-2,686.26$ & 4.0000 \\
\hline $3 d_{5 / 2}$ & $-2,187.19$ & 6.0000 & $-5,156.31$ & 1.4756 & $-2,622.14$ & 6.0000 \\
\hline $4 s$ & -943.61 & 1.9899 & $-2,992.50$ & 0.0722 & $-1,341.97$ & 1.9793 \\
\hline $4 p_{1 / 2}$ & -846.60 & 1.9737 & $-2,917.45$ & 0.0672 & $-1,244.62$ & 1.9461 \\
\hline $4 p_{3 / 2}$ & -771.68 & 3.8903 & $-2,777.18$ & 0.1173 & $-1,164.57$ & 3.7675 \\
\hline
\end{tabular}


Table 8. Cont.

\begin{tabular}{|c|c|c|c|c|c|c|}
\hline \multirow{3}{*}{$\frac{\frac{\rho\left(\mathrm{g} / \mathrm{cm}^{3}\right)}{k_{\beta} T(\mathrm{eV})}}{\text { Shell }}$} & \multicolumn{4}{|c|}{1.93} & \multirow{2}{*}{\multicolumn{2}{|c|}{$\begin{array}{c}0.01 \\
100\end{array}$}} \\
\hline & \multicolumn{2}{|c|}{100} & \multicolumn{2}{|c|}{1000} & & \\
\hline & $\varepsilon_{i}(\mathbf{e V})$ & $N_{i}$ & $\varepsilon_{i}(\mathbf{e V})$ & $N_{i}$ & $\varepsilon_{i}(\mathrm{eV})$ & $N_{i}$ \\
\hline $4 d_{3 / 2}$ & -611.08 & 3.5074 & $-2,675.58$ & 0.1063 & $-1,003.80$ & 3.0579 \\
\hline $4 d_{5 / 2}$ & -596.36 & 5.1603 & $-2,641.73$ & 0.1542 & -987.58 & 4.4041 \\
\hline $4 f_{5 / 2}$ & -393.86 & 2.6872 & $-2,559.40$ & 0.1423 & -788.06 & 1.6374 \\
\hline $4 f_{7 / 2}$ & -390.46 & 3.5159 & $-2,545.99$ & 0.1873 & -784.05 & 2.1200 \\
\hline $5 s$ & -340.19 & 0.6434 & $-1,699.91$ & 0.0204 & -664.83 & 0.1973 \\
\hline $5 p_{1 / 2}$ & -301.56 & 0.4875 & $-1,661.98$ & 0.0196 & -622.67 & 0.1340 \\
\hline $5 p_{3 / 2}$ & -279.82 & 0.8237 & $-1,593.62$ & 0.0367 & -594.56 & 0.2057 \\
\hline $5 d_{3 / 2}$ & -217.64 & 0.4890 & $-1,542.96$ & 0.0349 & -525.70 & 0.1060 \\
\hline $5 d_{5 / 2}$ & -213.51 & 0.7072 & $-1,526.14$ & 0.0514 & -519.92 & 0.1503 \\
\hline $5 f_{5 / 2}$ & -141.06 & 0.3649 & $-1,485.69$ & 0.0494 & -438.97 & 0.0678 \\
\hline $5 f_{7 / 2}$ & -140.15 & 0.4824 & $-1,478.91$ & 0.0654 & -437.49 & 0.0891 \\
\hline $5 g_{7 / 2}$ & -81.19 & 0.2749 & $-1,453.32$ & 0.0638 & -372.22 & 0.0467 \\
\hline $5 g_{9 / 2}$ & -81.09 & 0.3432 & $-1,449.76$ & 0.0795 & -371.92 & 0.0582 \\
\hline $6 s$ & -138.52 & 0.1188 & $-1,033.83$ & 0.0105 & -393.88 & 0.0145 \\
\hline $6 p_{1 / 2}$ & -121.22 & 0.1008 & $-1,012.24$ & 0.0103 & -372.63 & 0.0117 \\
\hline $6 p_{3 / 2}$ & -112.55 & 0.1857 & -974.08 & 0.0198 & -359.21 & 0.0205 \\
\hline $6 d_{3 / 2}$ & -84.58 & 0.1420 & -945.29 & 0.0193 & -324.12 & 0.0145 \\
\hline $6 d_{5 / 2}$ & -82.89 & 0.2096 & -935.78 & 0.0286 & -321.26 & 0.0211 \\
\hline $6 f_{5 / 2}$ & -50.56 & 0.1531 & -912.78 & 0.0280 & -280.20 & 0.0140 \\
\hline $6 f_{7 / 2}$ & -50.17 & 0.2034 & -908.90 & 0.0371 & -279.43 & 0.0185 \\
\hline $7 s$ & -52.94 & 0.0522 & -647.85 & 0.0072 & -256.64 & 0.0037 \\
\hline $7 p_{1 / 2}$ & -44.39 & 0.0481 & -634.47 & 0.0071 & -244.52 & 0.0033 \\
\hline $7 p_{3 / 2}$ & -40.42 & 0.0925 & -611.16 & 0.0138 & -237.03 & 0.0061 \\
\hline $7 d_{3 / 2}$ & -26.66 & 0.0808 & -593.27 & 0.0136 & -216.78 & 0.0050 \\
\hline $7 d_{5 / 2}$ & -25.89 & 0.1203 & -587.41 & 0.0202 & -215.14 & 0.0073 \\
\hline$N_{\text {bound }}$ & & 56.8484 & & 16.9143 & & 48.1074 \\
\hline$q$ & & 17.1516 & & 57.0857 & & 25.8926 \\
\hline$\mu(\mathrm{eV})$ & -414.80 & & $-6,276.71$ & & -886.06 & \\
\hline
\end{tabular}

To study tungsten impurities in fusion plasmas, we use the non-linear SCF screening model [39,40] for the calculation of the screening impurity potential. In the model, impurities in plasmas are considered as neutral pseudo-atoms. $R_{\mathrm{WS}}$ is assumed to be large. The chemical potential $\mu$ is found before the SCF calculations on the basis of the prescribed values of the plasmas electron density $N_{e}$ and temperature $k_{\beta} T$ using the following expression [40]:

$$
N_{e}=\frac{\sqrt{2}}{\pi^{2}}\left(k_{\beta} T\right)^{3 / 2} \int_{0}^{\infty} \frac{y^{1 / 2} d y}{1+\exp \left(y-\mu / k_{\beta} T\right)}
$$


We consider the typical fusion plasmas density $N_{e}=10^{14} \mathrm{~cm}^{-3}$ and the low temperature range $1 \mathrm{eV} \leq k_{\beta} T \leq 5 \mathrm{eV}$. The SCF potential $V(r)$ and the total electron density $\rho(r)$ are found by the iterative process described above. The DS-DS model is used.

In Figure 14, the ion charge $q$ (14a) and the chemical potential $\mu$ (14b) are displayed. As is evident, values of the charge and chemical potential change noticeably when the temperature increases. In Table 9, we present spectra obtained for the tungsten ion at various values of temperature. The case $k_{\beta} T=0$ refers to the usual DS calculations for a free neutral tungsten atom. One can see that the increasing of temperature causes all binding energies of inner levels to become lower by approximately the same value. Outer levels also become lower, relatively to a greater extent. Consequently, the energy spectrum depends considerably on a plasma temperature, the changes being different for inner and outer levels. Calculations showed that only valence $5 d_{3 / 2}$ and $5 d_{5 / 2}$ states have large occupation numbers, while other excited states involved in calculations with regard to a temperature, for example $5 f, 6 d, 6 f$, $7 d, 7 s$ and $7 p$, have zero occupation numbers. The $6 s$ and especially $6 p$ states have very small occupation numbers $(\lesssim 0.1)$, which decrease when temperature increases.

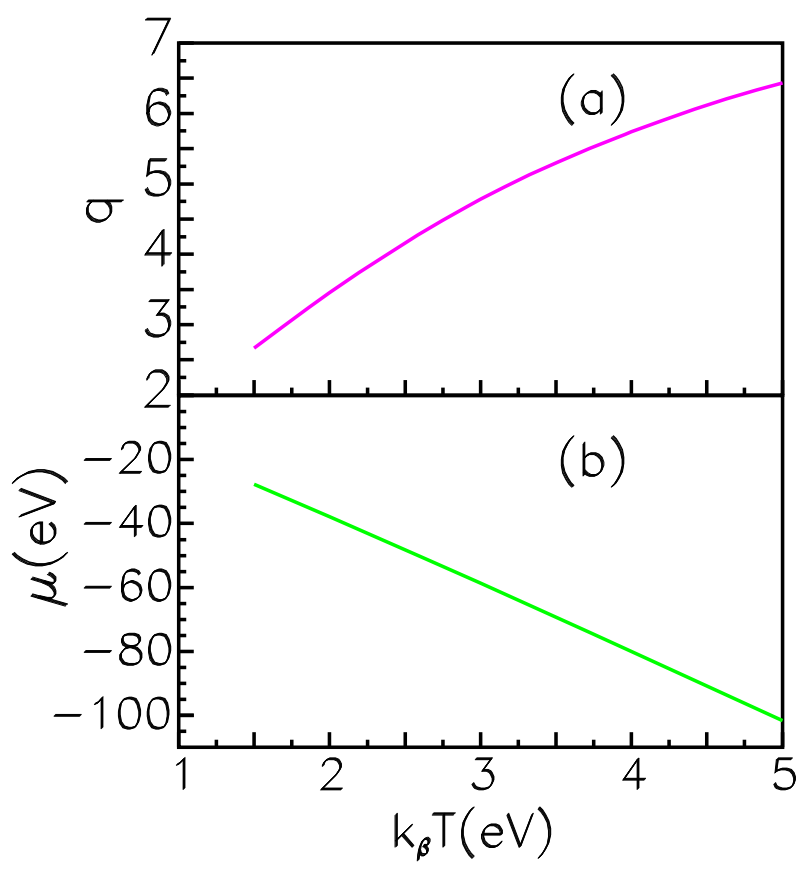

Figure 14. The charge $q$ (a) and the chemical potential $\mu$ (b) for the impurity tungsten ion in fusion plasmas.

The data of Table 9 demonstrate different results for the free neutral atom $\left(k_{\beta} T=0\right)$ and for calculations with regard to a temperature. Nevertheless, this was just calculations for a free neutral atom, which were adopted as the initial data, as for instance in [41], where the non-LTE calculations in the collisional radiative model were performed. The average ionization stage $\langle q\rangle=2.07$ was obtained in [41] for the tungsten ion at the electron density $N_{e}=10^{14} \mathrm{~cm}^{-3}$ and $k_{\beta} T=2 \mathrm{eV}$. It was also shown that the largest contributions were made by transitions $5 d^{3} 6 s^{1} \rightarrow 5 d^{3} 6 p^{1}$ and $5 d^{4} \rightarrow 5 d^{3} 6 p^{1}$. This means that the $5 d, 6 s$ and $6 p$ states are of primary importance in [41] as in our calculations. We obtained the ionization stage $q=3.45$. Therefore, we believe that our results could be used as initial data in more 
sophisticated calculations rather than data for a free neutral atom. This may considerably change the results of these calculations.

Table 9. Spectrum of energies $\varepsilon_{i}$ and level populations $N_{i}$ for the tungsten ion at low temperatures $\left(k_{\beta} T=2,3 \mathrm{eV}\right)$ as well as for a free neutral tungsten atom $\left(k_{\beta} T=0\right)$.

\begin{tabular}{|c|c|c|c|c|c|c|}
\hline \multirow{2}{*}{$\frac{k_{\beta} T(\mathrm{eV})}{\text { Shell }}$} & \multicolumn{2}{|l|}{0.0} & \multicolumn{2}{|c|}{2.0} & \multicolumn{2}{|c|}{3.0} \\
\hline & $\varepsilon_{i}(\mathrm{eV})$ & $N_{i}$ & $\varepsilon_{i}(\mathrm{eV})$ & $N_{i}$ & $\varepsilon_{i}(\mathbf{e V})$ & $N_{i}$ \\
\hline $1 s$ & $-69,312.37$ & 2.0 & $-69,346.96$ & 2.0000 & $-69,367.14$ & 2.0000 \\
\hline $2 s$ & $-11,956.28$ & 2.0 & $-11,990.83$ & 2.0000 & $-12,011.08$ & 2.0000 \\
\hline $2 p_{1 / 2}$ & $-11,439.93$ & 2.0 & $-11,474.51$ & 2.0000 & $-11,494.76$ & 2.0000 \\
\hline $2 p_{3 / 2}$ & $-10,088.80$ & 4.0 & $-10,123.37$ & 4.0000 & $-10,143.62$ & 4.0000 \\
\hline $3 s$ & -2752.49 & 2.0 & -2786.88 & 2.0000 & -2806.91 & 2.0000 \\
\hline $3 p_{1 / 2}$ & -2521.35 & 2.0 & -2555.74 & 2.0000 & -2575.79 & 2.0000 \\
\hline $3 p_{3 / 2}$ & -2229.29 & 4.0 & -2263.68 & 4.0000 & -2283.70 & 4.0000 \\
\hline $3 d_{3 / 2}$ & -1837.59 & 4.0 & -1872.00 & 4.0000 & -1892.07 & 4.0000 \\
\hline $3 d_{5 / 2}$ & -1774.01 & 6.0 & -1808.42 & 6.0000 & -1828.49 & 6.0000 \\
\hline $4 s$ & -566.71 & 2.0 & -601.09 & 2.0000 & -621.06 & 2.0000 \\
\hline $4 p_{1 / 2}$ & -470.77 & 2.0 & -505.15 & 2.0000 & -525.13 & 2.0000 \\
\hline $4 p_{3 / 2}$ & -402.85 & 4.0 & -437.23 & 4.0000 & -457.21 & 4.0000 \\
\hline $4 d_{3 / 2}$ & -244.71 & 4.0 & -279.10 & 4.0000 & -299.07 & 4.0000 \\
\hline $4 d_{5 / 2}$ & -232.20 & 6.0 & -266.58 & 6.0000 & -286.56 & 6.0000 \\
\hline $4 f_{5 / 2}$ & -34.17 & 6.0 & -68.50 & 6.0000 & -88.39 & 6.0000 \\
\hline $4 f_{7 / 2}$ & -31.93 & 8.0 & -66.26 & 8.0000 & -86.13 & 7.9992 \\
\hline $5 s$ & -78.80 & 2.0 & -112.82 & 2.0000 & -132.23 & 2.0000 \\
\hline $5 p_{1 / 2}$ & -50.29 & 2.0 & -84.06 & 2.0000 & -103.16 & 2.0000 \\
\hline $5 p_{3 / 2}$ & -40.40 & 4.0 & -73.90 & 4.0000 & -92.67 & 4.0000 \\
\hline $5 d_{3 / 2}$ & -5.10 & 4.0 & -36.20 & 1.2052 & -53.32 & 0.5788 \\
\hline $5 d_{5 / 2}$ & & & -35.20 & 1.2392 & -52.15 & 0.6149 \\
\hline $6 s$ & -6.35 & 2.0 & -31.99 & 0.0996 & -45.45 & 0.0242 \\
\hline $6 p_{1 / 2}$ & & & -25.14 & 0.0034 & -37.44 & 0.0017 \\
\hline $6 p_{3 / 2}$ & & & -23.47 & 0.0030 & -35.25 & 0.0016 \\
\hline$N_{\text {bound }}$ & & 74.0 & & 70.5504 & & 69.2204 \\
\hline$q$ & & 0.0 & & 3.4496 & & 4.7796 \\
\hline$\mu(\mathrm{eV})$ & & & -37.89 & & -58.66 & \\
\hline
\end{tabular}

\section{Conclusions}

- Our unified database on the RR and photoionization data was supplemented with partial and total RRCS and RR/RPL rates, as well as partial PCS for 54 tungsten ions from the range $\mathrm{W}^{6+}-\mathrm{W}^{71+}$. Fully relativistic calculations were performed by the DF method. All multipoles of a radiative field were taken into account. Total RRCS were calculated in the electron energy range from 
$1 \mathrm{eV}-\sim 80 \mathrm{keV}$. Partial PCS were fitted in a wide photon energy range by the analytical expression with five fit parameters for all electron states with $n \leq 10$ and $\ell \leq 4$. The fitting accuracy is usually better than 2\%. Partial RRCS may be found by the use of the fit parameters and the relationship between RRCS and PCS. The partial and total RR/RPL rates were calculated in the temperature range from $10^{4} \mathrm{~K}-10^{9} \mathrm{~K}$. Total RR rates were fitted by an analytical expression with four fit parameters. All results were added to the IAEA electronic database. The data are required for fusion studies, for example at the reactor ITER and devices ASDEX Upgrade and EBIT.

- The influence of the core electron polarization following the RR process on RRCS was investigated for the ion Fe XVII, as well as highly-charged tungsten ions. The inclusion of the PRR channel was shown to eliminate the puzzling discrepancy between experimental and theoretical values of EIECS for dominant X-ray lines from Fe XVII. It was found for highly-charged tungsten ions that the PRR enhancement factor may reach more than $15 \%$. The factor depends on the photon energy, the principal quantum number of polarized shells and the ion charge. However, the factor is practically independent of the state into which an electron is captured in the RR process.

- The effect of plasmas temperature and density on the electron structure of an ion in LTE plasmas was studied. For this purpose, the code PLASMASATOM was created on the basis of the average-atom model. The bound and continuum electron densities are calculated by the relativistic DS method. Our calculations for the iron and uranium ions in dense plasmas are in good agreement with previous results. In particular, our calculations of the Fe ion charge $q$ correlate well with the mean ionization stages $\langle q>$ obtained by collaboration OPAC using various codes. Our values of $q$ are in excellent agreement with the best data of $\langle q\rangle$ from the Opacity Project, the difference being in the range from $0.2 \%-4 \%$.

- New calculations for the tungsten ion in dense plasmas demonstrated the temperature and dense dependence of the energy spectrum and level populations in a wide temperature range. Calculations were also performed for the impurity tungsten ion in fusion plasmas at low temperature. Comparison of the results with previous non-LTE calculations for tungsten impurity atoms allow one to arrive at the conclusion that our results could be used as initial data in more sophisticated calculations rather than data for a free neutral atom. This may change the results of these calculations.

\section{Acknowledgment}

This work was supported through the International Atomic Energy Agency, Contract No. 16357/RBF, which is gratefully acknowledged. Malvina B. Trzhaskovskaya thanks the Russian Scientific Foundation, Grant No. 14-22-00281.

\section{Author Contributions}

Vladimir K. Nikulin set up and designed the problems under consideration; Malvina B. Trzhaskovskaya developed computer codes and performed calculations. Both authors analyzed the results obtained and wrote the paper. 


\section{Appendix}

Table A1. Radiative recombination rate coefficients for $\mathrm{W}^{6+}$ in $\mathrm{cm}^{3} \times \mathrm{s}^{-1}$. Presented for a value to its right is the decimal order, e.g., $8.42-13=8.42 \times 10^{-13}$.

\begin{tabular}{|c|c|c|c|c|c|c|c|c|c|c|c|}
\hline \multirow{2}{*}{ Shell } & \multicolumn{11}{|c|}{$\log _{10} T(K)$} \\
\hline & 4.0 & 4.5 & 5.0 & 5.5 & 6.0 & 6.5 & 7.0 & 7.5 & 8.0 & 8.5 & 9.0 \\
\hline $5 d_{3 / 2}$ & $8.42-13$ & $4.54-13$ & $2.26-13$ & $9.38-14$ & $3.30-14$ & $2.23-14$ & $2.36-14$ & $1.57-14$ & $6.29-15$ & $1.68-15$ & $3.16-16$ \\
\hline $5 d_{5 / 2}$ & & & & $1.24-13$ & & $3.26-14$ & & & & & \\
\hline $5 f_{5 / 2}$ & $.02-13$ & $1.01-13$ & $4.22-14$ & $1.34-14$ & $8.99-15$ & $1.21-14$ & $8.25-15$ & $3.08-15$ & $7.78-16$ & & \\
\hline $5 f_{7 / 2}$ & $.59-13$ & $1.29-13$ & $5.35-14$ & $1.70-14$ & $1.23-14$ & $1.66-14$ & $1.12-14$ & & $1.04-15$ & $2.04-16$ & \\
\hline $5 g_{7 / 2}$ & $.10-13$ & $5.35-14$ & $2.17-14$ & $6.90-15$ & $1.74-15$ & $3.75-16$ & $7.35-17$ & $1.36-17$ & $2.40-18$ & $4.02-19$ & \\
\hline $5 g_{9 / 2}$ & $.38-13$ & $6.73-14$ & $2.73-14$ & $8.67-15$ & $2.19-15$ & $4.73-16$ & $9.26-17$ & $1.71-17$ & $3.03-18$ & $5.07-19$ & -20 \\
\hline $6 s_{1 / 2}$ & $83-14$ & $1.07-14$ & $6.75-15$ & $79-15$ & $3.94-15$ & $3.62-15$ & $.35-15$ & $2.77-15$ & $1.89-15$ & $.96-16$ & -16 \\
\hline $6 p_{1 / 2}$ & & & & & & $2.61-15$ & & & & & \\
\hline $6 p_{3 / 2}$ & $59-14$ & $4.73-14$ & $2.46-14$ & $1.26-$ & $7.61-15$ & $6.71-15$ & $61-15$ & & & & $.74-16$ \\
\hline $6 d_{3 / 2}$ & $56-13$ & $1.43-13$ & $7.02-14$ & $2.86-14$ & $9.62-15$ & $5.72-15$ & & $3.92-15$ & & & $92-17$ \\
\hline $6 d_{5 / 2}$ & $78-13$ & $2.02-13$ & $9.87-14$ & $3.97-14$ & $1.35-14$ & $8.59-15$ & & & & & $.07-16$ \\
\hline $6 f_{5 / 2}$ & & $9.01-14$ & $3.82-14$ & 14 & & $7.32-15$ & & & & & \\
\hline $6 f_{7 / 2}$ & & & $4.88-14$ & & & $9.96-15$ & & & & & \\
\hline $6 g_{7 / 2}$ & & & & & & $4.29-1$ & & & & & \\
\hline $6 g_{9 / 2}$ & $1.57-13$ & $7.65-14$ & $3.11-14$ & $9.90-$ & $2.50-15$ & $5.40-16$ & $1.06-16$ & $1.95-$ & $3.46-18$ & $5.80-19$ & 8.51 \\
\hline $7 s_{1 / 2}$ & & & & & & $1.48-1$ & & & & & \\
\hline $7 p_{1 / 2}$ & & & & & & $1.20-1$ & & & & & \\
\hline $7 p_{3 / 2}$ & & & & & & $3.04-15$ & & & & & \\
\hline $7 d_{3 / 2}$ & & & & & & $2.84-15$ & & & & & \\
\hline $7 d_{5 / 2}$ & & & & & & & & & & & \\
\hline $7 f_{5 / 2}$ & & & & & & & & & & & \\
\hline $7 f_{7 / 2}$ & & & & & & & & & & & \\
\hline $7 g_{7 / 2}$ & & & & & & & & & & & \\
\hline $7 g_{9 / 2}$ & $1.37-13$ & $6.65-14$ & $2.71-14$ & $8.64-15$ & $2.18-15$ & $4.71-16$ & $9.23-17$ & $1.71-17$ & $3.02-18$ & -19 & $1.4 \mathrm{~J}$ \\
\hline $8 s_{1 / 2}$ & & & & & & $7.73-16$ & & & & & \\
\hline $8 p_{1 / 2}$ & & & & & & & & & & & \\
\hline $8 p_{3 / 2}$ & & & & & & & & & & & \\
\hline $8 d_{3 / 2}$ & & & & & & & & & & & \\
\hline $8 d_{5 / 2}$ & & $7.31-14$ & $50-14$ & & & 2.50 & & & & & \\
\hline $8 f_{5 / 2}$ & & $4.88-14$ & $2.09-14$ & & & $2.90-$ & & & & & \\
\hline $8 f_{7 / 2}$ & & & & & & $3.92-$ & & & & & \\
\hline $8 g_{7 / 2}$ & & $4.31-14$ & & & & $3.04-16$ & & & & & \\
\hline $8 g_{9 / 2}$ & $1.11-13$ & $5.40-14$ & $2.20-14$ & $7.01-15$ & $1.77-15$ & $3.82-16$ & $7.48-17$ & $1.38-17$ & $2.47-18$ & $4.19-19$ & $6.21-20$ \\
\hline $9 s_{1 / 2}$ & & & & & & $4.55-16$ & & & & & \\
\hline $9 p_{1 / 2}$ & & & & & & & & & & & \\
\hline $9 p_{3 / 2}$ & & & & & & & & & & & \\
\hline $9 d_{3 / 2}$ & & & & & & & & & & $7.23-17$ & \\
\hline $9 d_{5 / 2}$ & & & & & $2.92-15$ & $1.59-15$ & & & & $1.01-16$ & $1.86-17$ \\
\hline $9 f_{5 / 2}$ & & & & & & $1.97-15$ & & & & & \\
\hline $9 f_{7 / 2}$ & & $4.69-14$ & & & & & & & & & $5.17-18$ \\
\hline $9 g_{7 / 2}$ & 24 & $3.43-14$ & & $4.43-15$ & & $2.41-16$ & & & & & \\
\hline $9 g_{9 / 2}$ & $8.78-14$ & $4.29-14$ & $1.75-14$ & $5.56-15$ & $1.41-15$ & $3.03-1$ & $5.95-17$ & $1.11-17$ & $1.99-18$ & $3.36-19$ & $4.94-20$ \\
\hline $10 s_{1 / 2}$ & & & & & & & & & & & \\
\hline $10 p_{1 / 2}$ & & & & & & & & & & & \\
\hline $10 p_{3 / 2}$ & $1.86-14$ & $9.46-15$ & & $1.83-15$ & & $6.58-16$ & $6.18-16$ & & $2.66-16$ & & $2.55-17$ \\
\hline $10 d_{3 / 2}$ & $4.50-14$ & $2.37-14$ & $1.13-14$ & $4.40-15$ & $1.39-15$ & $7.05-16$ & $6.75-16$ & $4.48-16$ & $1.80-16$ & $4.83-17$ & $9.07-18$ \\
\hline $10 d_{5 / 2}$ & & $3.52-14$ & & & $2.02-15$ & $1.07-15$ & $1.02-15$ & $6.55-16$ & $2.56-16$ & $6.73-17$ & $1.24-17$ \\
\hline $10 f_{5 / 2}$ & & & & & & & $9.49-16$ & & & & \\
\hline $10 f_{7 / 2}$ & & & & & & $1.89-1$. & $1.27-15$ & & $1.20-16$ & $2.35-17$ & $3.66-18$ \\
\hline $10 g_{7 / 2}$ & & & & & & $1.94-16$ & $3.80-17$ & & $1.24-18$ & $2.08-19$ & $3.05-20$ \\
\hline $10 g_{9 / 2}$ & $6.98-14$ & $3.40-14$ & $1.39-14$ & $4.41-15$ & $1.11-15$ & $2.41-16$ & $4.76-17$ & $8.94-18$ & $1.60-18$ & $2.69-19$ & $3.96-20$ \\
\hline Total & $2.13-11$ & $7.34-12$ & $2.66-12$ & $8.81-13$ & $3.04-13$ & $2.11-13$ & $1.77-13$ & $1.03-13$ & $4.22-14$ & $1.30-14$ & $3.03-15$ \\
\hline
\end{tabular}


Table A2. Radiated power loss rate coefficients for $\mathrm{W}^{6+}$ in Watts $\times \mathrm{cm}^{3}$. Presented for a value to its right is the decimal order, e.g., $8.75-30=8.75 \times 10^{-30}$.

\begin{tabular}{|c|c|c|c|c|c|c|c|c|c|c|c|}
\hline \multirow{2}{*}{ Shell } & \multicolumn{11}{|c|}{$\log _{10} T(K)$} \\
\hline & 4.0 & 4.5 & 5.0 & 5.5 & 6.0 & 6.5 & 7.0 & 7.5 & 8.0 & 8.5 & 9.0 \\
\hline $5 d_{3 / 2}$ & $8.75-30$ & $4.84-30$ & & $1.22-30$ & $6.56-31$ & $1.76-30$ & $4.78-30$ & $6.47-30$ & $4.82-30$ & $2.20-30$ & $6.48-31$ \\
\hline $5 d_{5 / 2}$ & & & & & & $2.62-30$ & & & & & \\
\hline $5 f_{5 / 2}$ & $3-30$ & $5.42-31$ & $2.46-31$ & & & $8.47-31$ & & & & & \\
\hline $5 f_{7 / 2}$ & $2-30$ & $6.92-31$ & & $1.26-31$ & $3.76-31$ & $1.16-30$ & & & & & \\
\hline $5 g_{7 / 2}$ & & & & & $1.09-32$ & $3.14-33$ & $8.09-34$ & & & & \\
\hline $5 g_{9 / 2}$ & $.53-31$ & $2.36-31$ & $1.09-31$ & $4.23-32$ & $1.37-32$ & $3.96-33$ & $1.02-33$ & $2.31-34$ & $4.57-35$ & & $1.20-36$ \\
\hline $6 s_{1 / 2}$ & $1.60-31$ & $9.75-32$ & $80 \quad 32$ & $6.78-$ & $1.10-31$ & $2.60-31$ & $6.77-31$ & $1.56-30$ & $2.87-30$ & $3.95-30$ & $3.76-30$ \\
\hline $6 p_{1 / 2}$ & & & & & & & & & & & \\
\hline $6 p_{3 / 2}$ & & & & & & $4.87-3$ & & & & & \\
\hline $6 d_{3 / 2}$ & So & & & & & $4.03-31$ & & & & & \\
\hline $6 d_{5 / 2}$ & 630 & & & & & $6.16-31$ & & & & & \\
\hline $6 f_{5 / 2}$ & & & & & & $4.98-31$ & & & & & \\
\hline $6 f_{7 / 2}$ & & & & & & $6.73-31$ & & & & & \\
\hline $6 g_{7 / 2}$ & $01 \quad 21$ & & & & & & & & & & \\
\hline $6 g_{9 / 2}$ & $3.64-31$ & $1.95-31$ & $9.47-32$ & $3.88-32$ & $1.33-32$ & $4.01-3$ & $1.07-33$ & $2.47-34$ & $4.93-$ & -36 & -36 \\
\hline $7 s_{1 / 2}$ & & & & & & & & & & & \\
\hline $7 p_{1 / 2}$ & & & & & & & & & & & \\
\hline $7 p_{3 / 2}$ & & & & & & & & & & & \\
\hline $7 d_{3 / 2}$ & & & & & & & & & & & \\
\hline $7 d_{5 / 2}$ & & & & & & & & & & & \\
\hline $7 f_{5 / 2}$ & & & & & & & & & & & \\
\hline $7 f_{7 / 2}$ & & & & & & & & & & & \\
\hline $7 g_{7 / 2}$ & 1 & & & & & & & & & & \\
\hline $7 g_{9 / 2}$ & $2.37-31$ & $1.31-31$ & $6.69-32$ & $2.89-32$ & $1.03-32$ & $3.23-33$ & & & & & \\
\hline $8 s_{1 / 2}$ & $1.38-32$ & & & & & $4.91-32$ & & & & & \\
\hline $8 p_{1 / 2}$ & & & & & & & & & & & \\
\hline $8 p_{3 / 2}$ & & & & & & & & & & & \\
\hline $8 d_{3 / 2}$ & & & & & & & & & & & \\
\hline $8 d_{5 / 2}$ & 21 & $1.89-31$ & & & & $1.62-31$ & & & & & $6-32$ \\
\hline $8 f_{5 / 2}$ & 60 & $9.72-32$ & $5.24-32$ & & $5.46-32$ & $1.89-31$ & & & & & $2.64-33$ \\
\hline $8 f_{7 / 2}$ & & $1.26-31$ & $6.72-32$ & & $7.50-32$ & & $3.24-31$ & $1.99-31$ & & & $3.36-33$ \\
\hline $8 g_{7 / 2}$ & (1) & $6.85-32$ & & & & & & $1.74-$ & & & $8.79-36$ \\
\hline $8 g_{9 / 2}$ & $1.51-31$ & $8.57-32$ & $4.59-32$ & $2.08-32$ & $7.71-33$ & & $6.91-34$ & $1.83-34$ & $6.29-35$ & $2.35-35$ & $2.63-36$ \\
\hline $9 s_{1 / 2}$ & & & & & & & & & & & \\
\hline $9 p_{1 / 2}$ & & & & & & & & & & & \\
\hline $9 p_{3 / 2}$ & & & & & & & & & & & $2.07-31$ \\
\hline $9 d_{3 / 2}$ & $10 \Omega$ & & & & & & & & & & \\
\hline $9 d_{5 / 2}$ & $1.63-31$ & $9.90-32$ & $6.29-32$ & & & & & & & & $3.48-32$ \\
\hline $9 f_{5 / 2}$ & $9.73-32$ & $5.80-32$ & $3.28-32$ & & & & & & & & $1.79-33$ \\
\hline $9 f_{7 / 2}$ & $1.27-31$ & & & & & & & & & & $2.27-33$ \\
\hline $9 g_{7 / 2}$ & $7.74-32$ & $4.55-32$ & $2.55-32$ & & & & $4.82-34$ & & & & $1.36-36$ \\
\hline $9 g_{9 / 2}$ & $9.68-32$ & $5.70-32$ & $3.19-32$ & $1.50-32$ & $5.75-33$ & $1.89-3$ & $5.70-$ & $1.84-34$ & & $9.51-36$ & $1.48-36$ \\
\hline $10 s_{1 / 2}$ & & & & & & & & & & & \\
\hline $10 p_{1 / 2}$ & & $1.30-32$ & & & $5.68-33$ & & $5.02-32$ & & & & $1.07-31$ \\
\hline $10 p_{3 / 2}$ & & $1.71-32$ & $1.08-32$ & $8.68-33$ & $1.38-32$ & $4.17-32$ & $1.21-31$ & & & & $1.34-31$ \\
\hline $10 d_{3 / 2}$ & $6.13-32$ & $3.86-32$ & $2.61-32$ & $1.64-32$ & $1.18-32$ & $4.29-32$ & $1.29-31$ & & & $6.29-32$ & $1.86-32$ \\
\hline $10 d_{5 / 2}$ & $9.11-32$ & $5.70-32$ & $3.80-32$ & $2.34-32$ & $1.78-32$ & $6.67-32$ & $1.91-31$ & $2.58-31$ & $1.88-31$ & $8.26-32$ & $2.32-32$ \\
\hline $10 f_{5 / 2}$ & & $3.66-32$ & & $1.02-32$ & & & & & & & $1.27-33$ \\
\hline $10 f_{7 / 2}$ & $7.79-32$ & $4.77-32$ & $2.79-32$ & $1.31-32$ & $3.46-32$ & $1.20-31$ & $1.55-31$ & $9.54-32$ & $3.52-32$ & $8.89-33$ & $1.62-33$ \\
\hline $10 g_{7 / 2}$ & $5.12-32$ & $3.11-32$ & $1.82-32$ & $8.85-33$ & & $1.09-33$ & $2.59-34$ & $5.11-35$ & $9.22-36$ & $1.55-36$ & $2.28-37$ \\
\hline $10 g_{9 / 2}$ & $6.39-32$ & $3.89-32$ & $2.28-32$ & $1.11-32$ & $4.35-33$ & & $5.11-34$ & $1.53-34$ & $3.38-35$ & $6.11-36$ & $9.19-37$ \\
\hline Total & $4.12-29$ & $2.20-29$ & $1.15-29$ & $5.55-30$ & $4.82-30$ & $1.41-29$ & $3.05-29$ & $4.02-29$ & $3.65-29$ & $2.60-29$ & $1.52-29$ \\
\hline
\end{tabular}

\section{Conflicts of Interest}

There are no conflicts of interest. 


\section{References}

1. Neu, R.; Dux, R.; Kallenbach, A.; Pütterich, T.; Balden, M.; Fuchs, J.C.; Herrmann, A.; Maggi, C.F.; O’Mullane, M.; Pugno, R.; et al. Tungsten: An option for divertor and main chamber plasma facing components in future fusion devices. Nucl. Fusion 2005, 45, 209-218.

2. Reader, J. Spectral data for fusion energy: From W to W. Phys. Scr. 2009, 2009, 014023.

3. Skinner, C.H. Atomic physics in the quest for fusion energy and ITER. Phys. Scr. 2009, 2009, 014022 .

4. Biedermann, C.; Radtke, R.; Seidel, R.; Pütterich, T. Spectroscopy of highly charged tungsten ions relevant to fusion plasmas. Phys. Scr. 2009, 2009, 014026.

5. Trzhaskovskaya, M.B.; Nikulin, V.K.; Clark, R.E.H. Radiative recombination and photoionization cross sections for heavy element impurities in plasmas. At. Data Nucl. Data Tables 2008, 94, 71-139.

6. Trzhaskovskaya, M.B.; Nikulin, V.K.; Clark, R.E.H. Radiative recombination rate coefficients for highly-charged tungsten ions. At. Data Nucl. Data Tables 2010, 96, 1-25.

7. Trzhaskovskaya, M.B.; Nikulin, V.K.; Clark, R.E.H. Horizons in World Physics; Reimer, A., Ed.; Nova: New York, NY, USA, 2012; Volume 277, Chapter 8, pp. 173-229.

8. Trzhaskovskaya, M.B.; Nikulin, V.K. Radiative recombination data for tungsten ions: I. $\mathrm{W}^{24+}-\mathrm{W}^{45+}$. At. Data Nucl. Data Tables 2013, 99, 249-311.

9. Trzhaskovskaya, M.B.; Nikulin, V.K. Radiative recombination data for tungsten ions: II. $\mathrm{W}^{47+}-\mathrm{W}^{71+}$. At. Data Nucl. Data Tables 2014, 100, 986-1058.

10. Trzhaskovskaya, M.B.; Nikulin, V.K. Radiative recombination data for tungsten ions: III. $\mathrm{W}^{14+}-\mathrm{W}^{23+}$. At. Data Nucl. Data Tables 2014, 100, 1156-1188.

11. Trzhaskovskaya, M.B.; Nikulin, V.K. Radiative Recombination and Photoionization Data of Heavy Element Impurities in Plasmas. Available online: http://www-amdis.iaea.org/ DATASETS/a74.php (accessed on 10 April 2015).

12. Nikulin, V.K.; Trzhaskovskaya, M.B. Comment on "Energy dependent excitation cross section measurements of diagnostic lines of Fe XVII". Phys. Rev. Lett. 2012, 108, 139301.

13. Brown, G.V.; Beiersdorfer, P.; Chen, H.; Scofield, J.H.; Boyce, K.R.; Kelley, R.L.; Kilbourne, C.A.; Porter, F.S.; Gu, M.F.; Kahn, S.M.; et al. Energy dependent excitation cross section measurements of diagnostic lines of Fe XVII. Phys. Rev. Lett. 2006, 96, 253201.

14. Trzhaskovskaya, M.B.; Nikulin, V.K. Polarization Radiative Recombination Effect for Tungsten Highly-Charged Ions; Petersburg Nuclear Physics Institute Report No PNPI-2893; Petersburg Nuclear Physics Institute: Gatchina, Russia, 2012; pp. 1-22.

15. Trzhaskovskaya, M.B.; Nikulin, V.K. Atom in Dense Laser and Fusion Plasmas; Petersburg Nuclear Physics Institute Report No PNPI-2932; Petersburg Nuclear Physics Institute: Gatchina, Russia, 2013; pp. 1-26.

16. Band, I.M.; Listengarten, M.A.; Trzhaskovskaya, M.B.; Fomichev, V.I. Computer Program Complex RAINE. Part I; Leningrad Nuclear Physics Institute Report No LNPI-289; Leningrad Nuclear Physics Institute: Gatchina, Russia, 1976; pp. 1-54. 
17. Band, I.M.; Trzhaskovskaya, M.B.; Fomichev, V.I. Computer Program Complex RAINE. Part III; Leningrad Nuclear Physics Institute Report No LNPI-299; Leningrad Nuclear Physics Institute: Gatchina, Russia, 1977; pp. 1-46.

18. Band, I.M.; Trzhaskovskaya, M.B.; Nestor, C.W., Jr.; Tikkanen, P.O.; Raman, S. Dirac-Fock internal conversion coefficients. At. Data Nucl. Data Tables 2002, 81, 1-334.

19. Liberman, D.A. Selfconsistent field for condensed matter. Phys. Rev. B 1979, 20, 4981-4989.

20. Bennet, B.I.; Liberman, D.A. Inferno; Los Alamos National Laboratory Report No LA-10309-M; Los Alamos National Laboratory: Los Alamos, NM, USA, 1985; pp. 1-32.

21. Wilson, B.; Sonnad, V.; Sterne, P.; Isaacs, W. Purgatorio-A new implementation of the Inferno algorithm. J. Quant. Spectrosc. Rad. Transfer. 2006, 99, 658-685.

22. Pénicaud, M. An average atom code for warm matter: Application to aluminium and uranium. J. Phys. 2009, 21, 095409.

23. Ichihara, A.; Eichler, J. Cross sections for radiative recombination and the photoelectric effect in the $\mathrm{K}, \mathrm{L}$, and $\mathrm{M}$ shells of one-electron systems with $1 \leq Z \leq 112$ calculated within an exact relativistic description. At. Data Nucl. Data Tables 2000, 74, 1-121.

24. Alexanian, M. Photon bremsstrahlung from an extreme-relativistic electron gas. Phys. Rev. 1968, $165,253-257$.

25. Trzhaskovskaya, M.B; Nikulin, V.K.; Clark R.E.H. Multipole and relativistic effects in radiative recombination process in hot plasmas. Phys. Rev. E 2008, 78, 035401(R).

26. Kramida, A.E.; Shirai, T. Energy levels and spectral lines of tungsten, W III through W LXXIV. At. Data Nucl. Data Tables 2009, 95, 305-474.

27. Verner, D.A.; Yakovlev, D.G.; Band, I.M.; Trzhaskovskaya, M.B. Subshell photoionization cross sections and ionization energies of atoms and ions from He to Zn. At. Data Nucl. Data Tables 1993, 55, 233-280.

28. Verner, D.A.; Ferland, G.J. Atomic data for astrophysics. I. Radiative recombination rates for H-like, He-like, Li-like, and Na-like ions over a broad range of temperature. Ap. J. Suppl. 1996, 103, 467-473.

29. Astapenko, V.A.; Bureeva, L.A.; Lisitsa, V.S. Statistical models in polarization radiation theory Radiat. Phys. Chem. 2006, 75, 1287-1307.

30. Badnell, N.R.; Pindzola, M.S. Unified dielectronic and radiative-recombination cross sections for $\mathrm{U}^{90+}$. Phys. Rev. A 1992, 45, 2820-2824.

31. Korol, A.V.; Gribakin, G.F.; Currell, F.J. The role of target polarization in electron-ion recombination. J. Phys. B 2004, 37, 2411-2428.

32. Korol, A.V.; Gribakin, G.F.; Currell, F.J. Effect of target polarization in electron-ion recombination. Phys. Rev. Lett. 2006, 97, 223201.

33. Kogan, V.I.; Kukushkin, A.B.; Lisitsa, V.S. Kramers electrodynamics and electron-atomic radiative-collisional processes. Phys. Rep. 1992, 213, 1-116.

34. Johnson, W.R. Photoabsorption in Hot Dense Plasmas. Available online: www3.nd.edu/ johnson/ Publications/ tdlda/pdf (accessed on 11 February 2001).

35. Johnson, W.R.; Guet, C.; Bertsch, G.F. Optical properties of plasma based on an average-atom model. J. Quant. Spectrosc. Rad. Transfer 2006, 99, 327-340. 
36. Blenski, T.; Ishikawa, K. Pressure ionization in the spherical ion-cell model of dense plasmas and a pressure formula in the relativistic Pauli approximation. Phys. Rev. E 1995, 51, 4869-4881.

37. Gilles, D.; Turck-Chieze, S.; Loisel, G.; Piau, L.; Ducret, J.-E.; Poirier, M.; Blenski, T.; Thais, F.; Blancard, C.; Cossé, P.; et al. Comparison of $\mathrm{Fe}$ and $\mathrm{Ni}$ opacity calculations for a better understanding of pulsating stellar envelopes. High Energy Dens. Phys. 2011, 7, 312-319.

38. Seaton, M.J.; Badnell, N.R. A comparison of Rosseland mean opacities from OP and OPAL. Mon. Not. R. Astron. Soc. 2004, 354, 457-465.

39. Dharma-Wardana, M.W.C.; Perrot, F. Level shift continuum lowering and the mobility edge in dense plasmas. Phys. Rev. A 1991, 45, 5883-5896.

40. Perrot, F. Temperature-dependent nonlinear screening of a photon in an electron gas. Phys. Rev. A 1982, 25, 489-495.

41. Abdallah, J., Jr.; Colgan, J.; Clark, R.E.H.; Fontes, C.J.; Zhang, H.L.J. A collisional-radiative study of low-temperature tungsten plasma model. J. Phys. B 2011, 44, 075701.

(c) 2015 by the authors; licensee MDPI, Basel, Switzerland. This article is an open access article distributed under the terms and conditions of the Creative Commons Attribution license (http://creativecommons.org/licenses/by/4.0/). 